\title{
The Population Structure and Selection Signal Analysis of Shenxian Pigs Based on Genome Resequencing Technology
}

Diao Liu

Agricultural University of Hebei

Chunlian Lu

Agricultural University of Hebei

Shang Li

Agricultural University of Hebei

Mengyu Jia

Agricultural University of Hebei

Yutao Miao

Hebei Veterinary Drug Supervision Institute

Liqin Ren

Hebei Fishery Technology Extension Station

Sai Li

Hebei Zhengnong Animal Husbandry Co., Ltd.

Hongzhan Cao ( $\nabla$ chz516@126.com )

Agricultural University of Hebei

\section{Research Article}

Keywords: Genome resequencing, Shenxian pig, SNP, Selective signal analysis

Posted Date: March 1st, 2021

DOI: https://doi.org/10.21203/rs.3.rs-244687/v1

License: (1) (1) This work is licensed under a Creative Commons Attribution 4.0 International License.

Read Full License 


\title{
The Population Structure and Selection Signal Analysis of Shenxian Pigs based on Genome Resequencing Technology
}

Diao Liu ${ }^{1}$, Chunlian $\mathrm{Lu}^{1}$, Shang $\mathrm{Li}^{1}$, Mengyu Jia ${ }^{1}$, Yutao Miao ${ }^{2}$, Liqin $\mathrm{Ren}^{3}$, Sai $\mathrm{Li}^{4}$ and Hongzhan $\mathrm{CaO}^{1 *}$

\begin{abstract}
Shenxian pigs are the only local black pig of Hebei Province, and were listed in the Genetics of Livestock and Poultry Resources of China in 2016. This breed of pig is considered to be a valuable local pig germplasm genetic resource in China. When compared with other introduced pig breeds, the Shenxian pig breed is characterized with early sexual maturity, short oestrus intervals, large litter sizes, and good meat quality, which are all of good research significance. However, the Shenxian pig variety was previously declared extinct in 2004 due to the introduction of a large number of foreign pig breeds. In order to preserve and study the Shenxian pig breed, the Hebei Zhengnong Animal Husbandry Co., Ltd. was established in Hebei Province for the purpose of preserving the purified Shenxian pig strain. In the present study, in order to understand the genetic variations of Shenxian pigs, identify selected regions related to superior traits, and accelerate the breeding processes of Shenxian pigs, the whole genome of the Shenxian pigs was resequenced and compared with that of large white pigs. The goal was to explore the germplasm characteristics of Shenxian pigs. The results obtained in this research investigation revealed that the genetic relationships of the Shenxian pig breed were complex, and that sub-populations could be identified within the general population. A total of 23M SNP sites were obtained by whole genome resequencing, and 1,509 selected sites were obtained via bioinformatics analyses. It was determined after annotation that a total of 19 genes were enriched in three items of bioengineering, molecular function, and cell composition.During this research investigation, the aforementioned 19 genes were subjected to GO and KEGG analyses. Subsequently, the candidate genes related to cell proliferation were obtained (DMTF1 and WDR5), which were considered to possibly be related to the slow growth and development of Shenxian pigs. In addition, the candidate genes related to lactation were obtained (CSN2 and CSN3). However, no genes related to meat quality traits were successfully screened.
\end{abstract}

\footnotetext{
${ }^{1}$ College of Animal Science and Technology, Hebei Agricultural University, Baoding 071000, Hebei.

${ }^{2}$ Hebei Veterinary Drug Supervision Institute, Shijiazhuang 050051, Hebei

${ }^{3}$ Fishery Technology Extension Station, Wanquan District, Zhangjiakou City, Zhangjiakou 076250, Hebei.

${ }^{4}$ Hebei Zhengnong Animal Husbandry Co., Ltd., Xinji 052360, Hebei.

* Corresponding author: E-mail: chz516@126. com.
} 
Keywords: Genome resequencing; Shenxian pig;SNP; Selective signal analysis

\section{Background}

China has a long history and rich experience in livestock and poultry breeding, and it is also one of the main domestication origins of pigs ${ }^{[1,2]}$. China has a vast territory, complex terrain, and rich pig resource pool. It can be seen that even the southern China pig breeds are quite different from the northern China ones ${ }^{[3]}$. As the only local black pig breed of Hebei Province, the Shenxian pig variety was declared extinct in 2004 due to the introduction of a large number of foreign pig breeds. Therefore, in order to protect and study the Shenxian pig breed, Hebei Zhengnong Animal Husbandry Co., Ltd. was established in Hebei Province, with the goal of preserving the purified Shenxian pig variety. Shenxian pigs were listed in the Genetics of Livestock and Poultry Resources of China in 2016. At the present time, a preserved population of Shenxian pigs has been developed, which consists of 28 boars and 220 sows. As a local breed in China, Shenxian pigs are known to have the excellent qualities of the majority of local pig breeds, with relatively strong adaptability to the environmental conditions and resistance to crude feed. The Shenxian pigs have been observed to have stronger reproductive capacities than introduced breeds, with the advantageous characteristics of early sexual maturity and larger litter sizes, and sows with strong lactation abilities. In addition, the breed is known for its higher intramuscular fat content and superior meat quality when compared with other domestic breeds.

At the present time, there have been few studies conducted regarding the molecular aspects of the Shenxian pig variety. In recent years, several research studies and tests have been carried out for Shenxian pigs using sequencing methods and other means. For example, using genome-wide association analyses of the main reproductive traits of Shenxian pigs, significant SNP sites which affect the reproductive traits of Shenxian pigs have been successfully screened. In addition, following various post-processing and software analysis processes, some significant SNP sites have been identified which affect the reproductive traits of the breed, and relevant target genes were obtained through site annotation. The previous related studies have identified TSKU, LRRC32, B3GNT6, WNT, LRRC32, CAPN5, MYO7A, TCHHL1, SI00A11, TACR3, IL12A, and THEM5 as the potential candidate genes which affect the reproductive performances of Shenxian pigs .

In the present study, based on newly developed resequencing technology, ten Shenxian pigs, nine large white pigs, and ten Meishan pigs were subjected to genetic analyses of population in order to obtain a systematic understanding regarding the population genetic relationships of Shenxian pigs. At the same time, a selection signal study was carried out with the large white pig genome, and selected regions in the genome were 
compared and analyzed. The goals were to deepen the current understanding of the selection differences between the Shenxian and large white pig breeds, as well as to identify the selection regions related to meat quality and reproductive traits, with the long-term goal of accelerating the breeding processes of the Shenxian pig variety.

\section{Results}

\subsection{Genome alignment and SNP identification}

\subsubsection{Reference genome alignment}

BWA software (version: 0.7.15-r1140) was adopted in this study to align the clean sequences of the Shenxian pig breed after quality control measures were implemented to the latest version of pig reference genome. The results are shown in Table 1. The results revealed that on average more than $86 \%$ of the data could be aligned to the reference genome.

Table 1. Statistics of the alignment results of the reference genomes

\begin{tabular}{ccccc}
\hline sample & Mapped & Paired & Insert & SD \\
\hline 1 & 86.75 & 76.36 & 387 & $'-128 /+129$ \\
2 & 86.47 & 74.70 & 408 & $'-129+134$ \\
3 & 87.12 & 75.61 & 407 & $'-124 /+124$ \\
4 & 87.09 & 76.07 & 415 & $'-126 /+124$ \\
5 & 87.01 & 75.91 & 365 & $'-110 /+114$ \\
6 & 86.11 & 75.40 & 399 & $'-120 /+115$ \\
7 & 85.02 & 73.37 & 396 & $'-122 /+122$ \\
8 & 87.15 & 75.39 & 386 & $'-118 /+135$ \\
9 & 87.04 & 75.15 & 436 & $'-141 /+140$ \\
10 & 86.66 & 75.44 & 380 & $'-114 /+120$ \\
\hline
\end{tabular}

\subsubsection{SNP identification}

Clean data were obtained after strict quality control and alignment processes of the obtained genome data were implemented. Then, GATK software was used to identify the SNP, and the SNP data were used for the subsequent analysis processes. A total of 34.2M SNP sites were identified in the examined Shenxian, large white, and Meishan pig individuals. There were determined to be 23M SNP sites in the Shenxian pig and 8.8M SNP sites in the large white pig varieties. In addition, there were 2.2M SNP sites in Meishan pig, and a total of 6.9M SNP sites in Shenxian pig and large white pig, respectively. This study found that more SNP sites had been identified in Shenxian pig strain, while a total of 1.5M SNP sites were identified in the Shenxian, large white, and 
Meishan pig varieties. It was possible that Shenxian pig breed had shown more genetic variations in the pig reference genome, while the Meishan and large white pig varieties had displayed less variability with the identified SNP sites when compared with the

Shenxian pig breed (Table 2). However, it was found to be difficult to extract common sites for analysis purposes, and the Meishan pigs were observed to be missing sites during the extraction processes of the common sites. Therefore, the subsequent population structure analyses and LD attenuation analyses were analyzed in pairs.

Table 2. Statistics of the SNP results

\begin{tabular}{cccc}
\hline Classification & Amount & Number of SNPs & Co-owned SNP \\
\hline Shenxian Pig & 10 & 23088823 & 1486285 \\
Large white pig & 9 & 8836786 & \\
Meishan pig & 10 & 2225776 & \\
\hline
\end{tabular}

\subsection{Population structure analyses}

\subsubsection{Principal component analyses}

In order to reveal the variety specificity of Shenxian pigs, principal component analyses (PCA) were performed on the obtained SNP information of the Shenxian, large white, and Meishan pig breeds, respectively, as shown in Figure 1 to Figure 3). Then, the first three eigenvectors were selected. The analysis results of the first eigenvector and the second eigenvector revealed that the first eigenvector had effectively distinguished the three breeds. The second eigenvector showed that the large white pig population was a population with small differentiations within the population. Meanwhile, population stratification was observed within the Shenxian and Meishan pig populations. The analysis results of the first eigenvector and the third eigenvector showed that the population stratification of the large white pig and Shenxian pig breeds was not obvious. However, the Meishan pig population displayed obvious stratification. The second eigenvector and the third eigenvector also revealed that there was a certain degree of stratification in the Meishan pig population. Furthermore, the results indicated that the stratification in the Meishan pig population was larger than that in both the Shenxian pig and large white pig populations. 

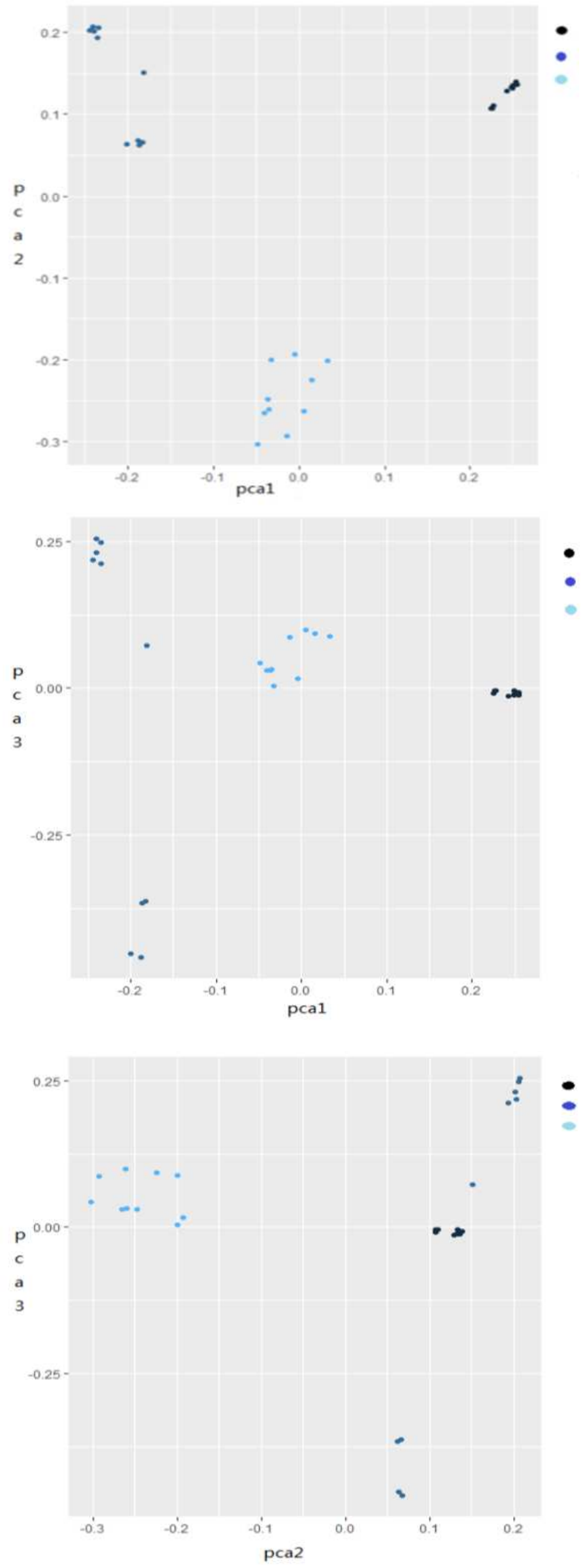
Note: Black represents big white pigs, dark blue represents Meishan pigs, light blue represents Shenxian pigs.

Figure 1 to Figure 3 Principal component analysis results

\subsubsection{Phylogenetic tree analyses}

In order to verify the results of the principal component analyses (PCA) among the Shenxian, large white, and Meishan pig breeds, the SNP data were used to construct the genetic distance matrixes of the three populations in plink software. Then, an evolutionary relationship analysis was carried out in order to draw the evolutionary tree, as shown in Figure 4. The analysis results showed that the three populations had originated from the same species. In addition, there were distant genetic relationships observed between the Shenxian pigs and the other two populations. However, when compared with the Shenxian pigs, the Meishan pigs were found to have a closer genetic relationship with the large white pigs.

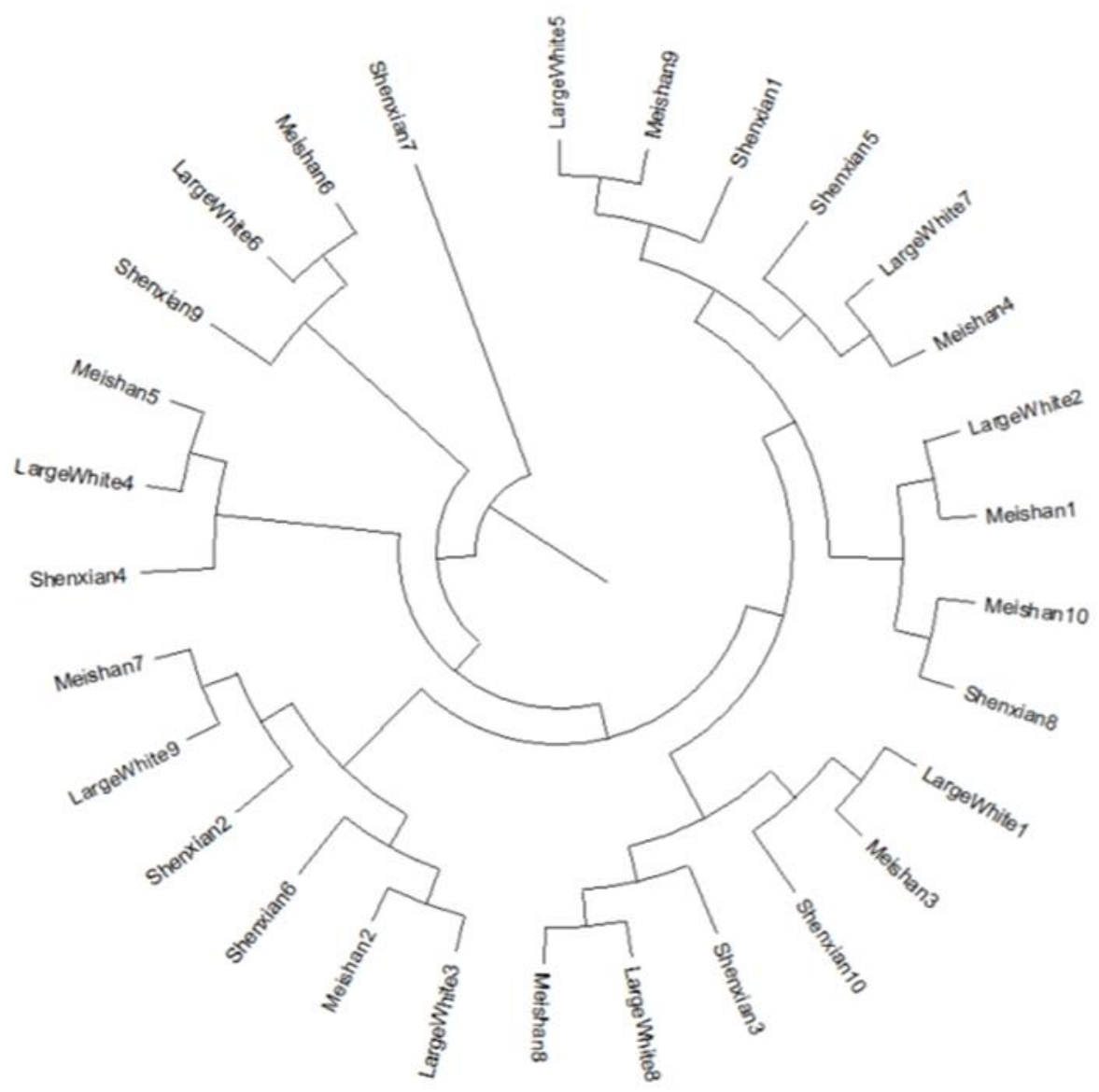

Fig. 2-2 Phylogenetic tree

\subsubsection{Population structure analyses}


Previous studies have shown that Asian breeds of pigs had completed domestication earlier than European breeds. However, due to geographic migrations, the genomes of European domesticated pigs are likely to include a large number of Asian pig genomes. In the current study, in order to explore the ancestral relationships of Shenxian pigs, the population structures of the Shenxian, large white, and Meishan pig varieties were further analyzed, as detailed in Figure 5 and Figure 6. The analysis results indicated that the large white pig variety was representative of the introduced breeds, and the Meishan pig variety was representative of the southern China pig breeds. It was observed that the degrees of intergenomic communication between the Shenxian pigs and the large white pigs were basically consistent with that between the Shenxian pigs and the Meishan pigs. However, when compared with the Meishan pig population, there were observed to be relatively more gene exchanges between the Shenxian and the large white pigs. These difference may have been caused by many factors, such as the infiltration of the Shenxian pig genes into European domesticated pigs after domestication was complete, or the introduction of the genome of the large white pig during the development and breed conservation of the Shenxian pig breed. Another possibility may have been the existence of common genomes between the two breeds.

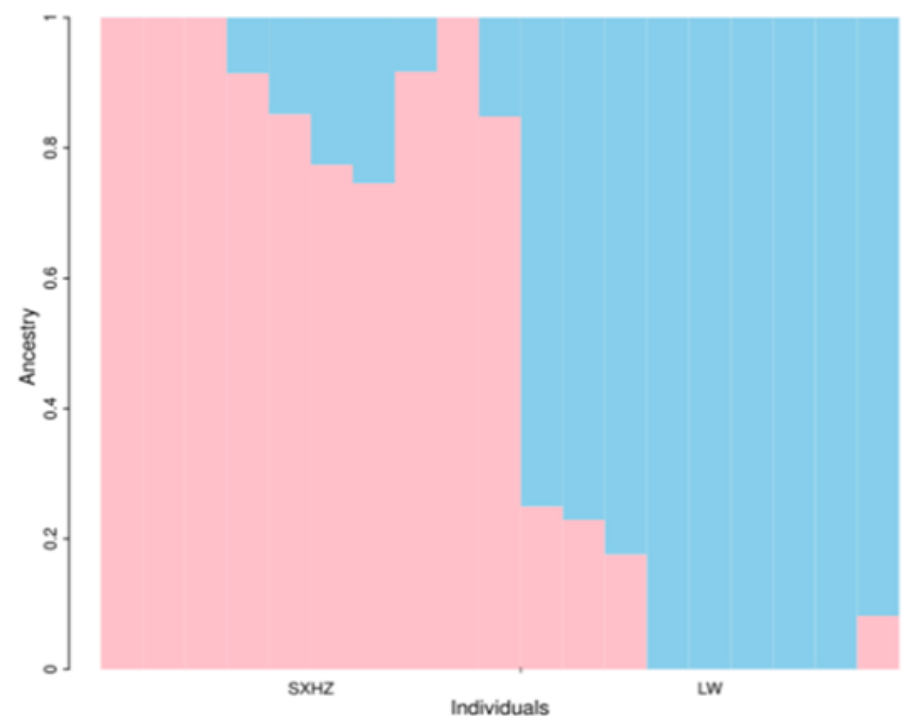

Note: The picture shows the group structure of Shenxian pigs and large white pigs. Pink means the Shenxian pigs, blue means the large white pigs. The abscissa represents the individuals in the group, and the ordinate represents the included ancestry.

Figure 5 Population structures of the Shenxian and large white pig breeds 


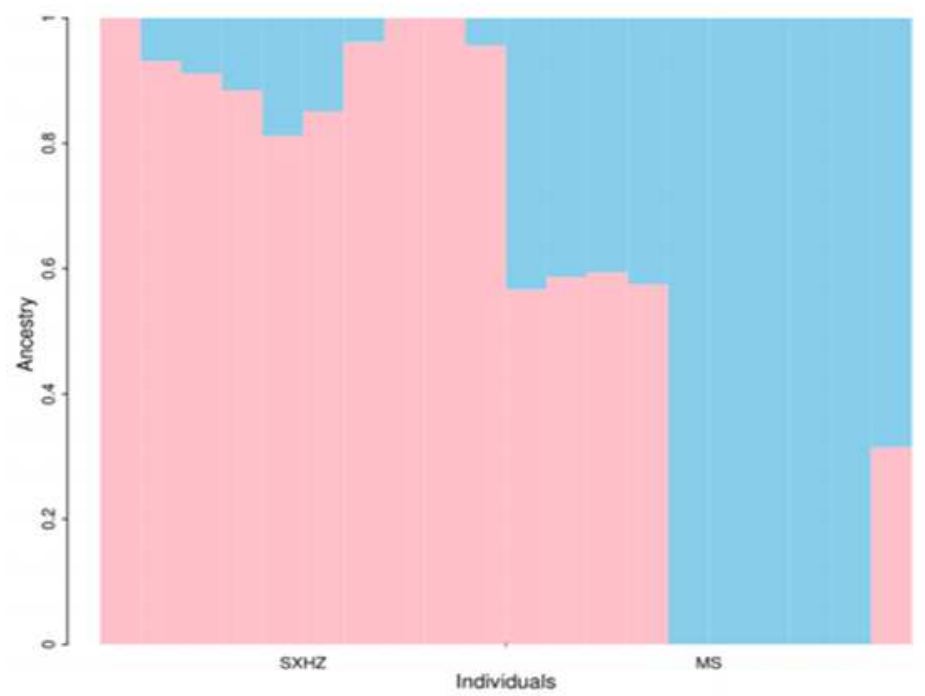

Note: The picture shows the group structure of Shenxian pigs and Meishan pigs. Pink means the Shenxian pigs, blue means the Meishan pigs in. The abscissa represents the individuals in the group, and the ordinate represents the included ancestry.

Figure 6 Population structures of the Shenxian and Meishan pig breeds

\subsubsection{Analysis results of the LD (linkage disequilibrium) attenuation}

The attenuation rates of the LD were found to be different among the different pig populations. It was found that the higher the selection intensity was, the slower the attenuation rate of the LD would be. The linkage disequilibrium of the Shenxian, large white, and Meishan pig populations was analyzed using PopLDdecay software. It was determined from the LD attenuation map that the linkage disequilibrium of the different populations decreased with the increases in marker spacings. The value of $\mathrm{D}$ was found to be strongly dependent on the artificial allele frequency, which was not conducive to a comparison of the LD attenuation. Therefore, the standardized disequilibrium coefficient $\mathrm{D}^{\prime}$ was adopted in order to avoid the dependence on the allele frequency.

The calculation method of $\mathrm{D}^{\prime}$ was as follows:

$$
\mathrm{D}^{\prime}=\mathrm{D} / \mathrm{Dmax}
$$

When $\mathrm{D}<0$, Dmax $=\min \{\mathrm{P}(\mathrm{A}) \mathrm{P}(\mathrm{B}), \mathrm{P}(\mathrm{a}) \mathrm{P}(\mathrm{b})\}$;

When $\mathrm{D}>0, \mathrm{Dmax}=\min \{\mathrm{P}(\mathrm{A}) \mathrm{P}(\mathrm{b}), \mathrm{P}(\mathrm{a}) \mathrm{P}(\mathrm{B})\}$;

When $\mathrm{D}^{\prime}=1$, it was indicated that the linkage was totally unbalanced and there was no reorganization;

Finally, when $\mathrm{D}^{\prime}=0$, it was indicated that the linkage was completely balanced with random reorganization. 
Generally speaking, the higher the domestication intensity was, the greater the selection intensity would be, and the smaller the LD attenuation rate would be. The results shown in the LD attenuation diagram (Figure 7 and Figure 8) revealed that the LD coefficient of the Shenxian pig population declined faster than that of the large white pig population. These findings indicated that the genetic diversity of the Shenxian pig population was higher.

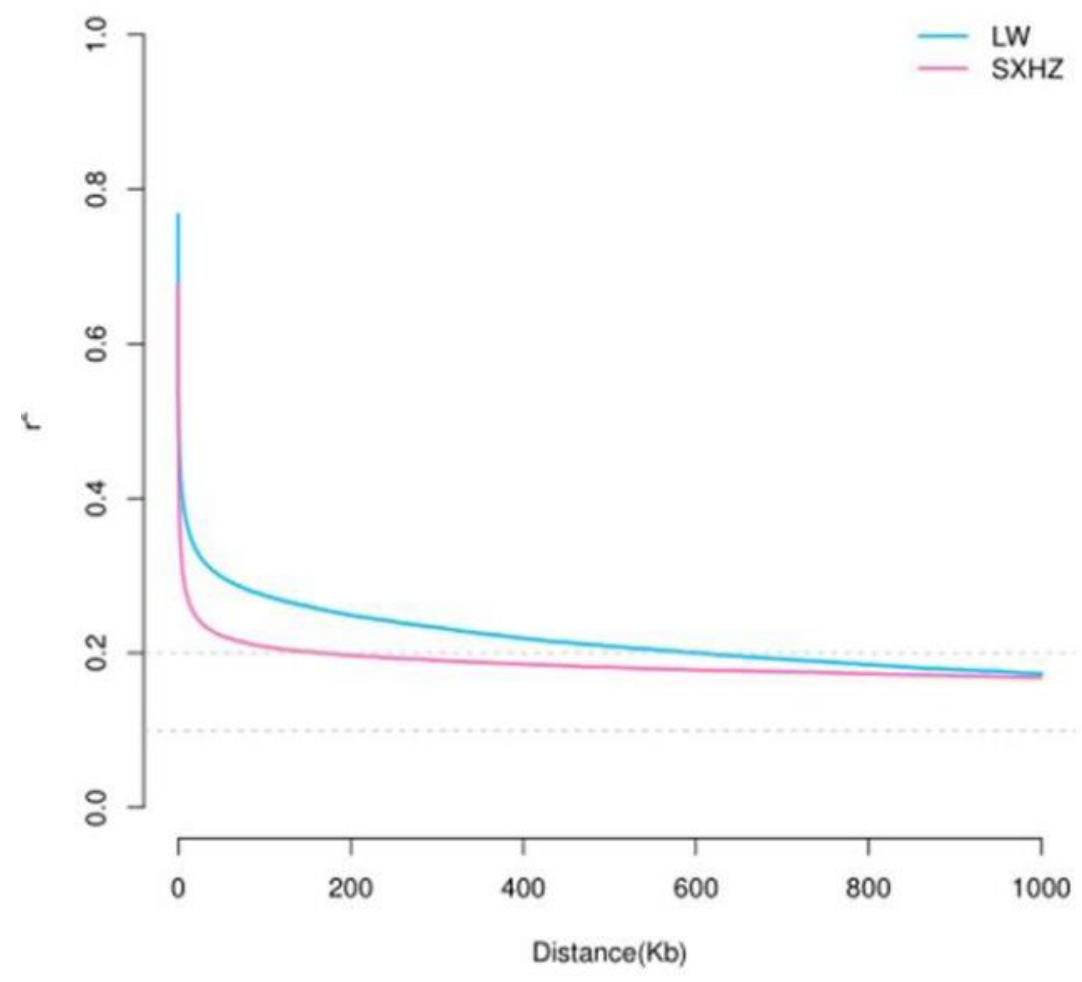

Note:The red line is the LD attenuation curve of Shenxian pigs, and the blue line is the LD attenuation curve of big white pigs.

Figure 7 LD attenuation diagram of the Shenxian pig and the large white pig populations 


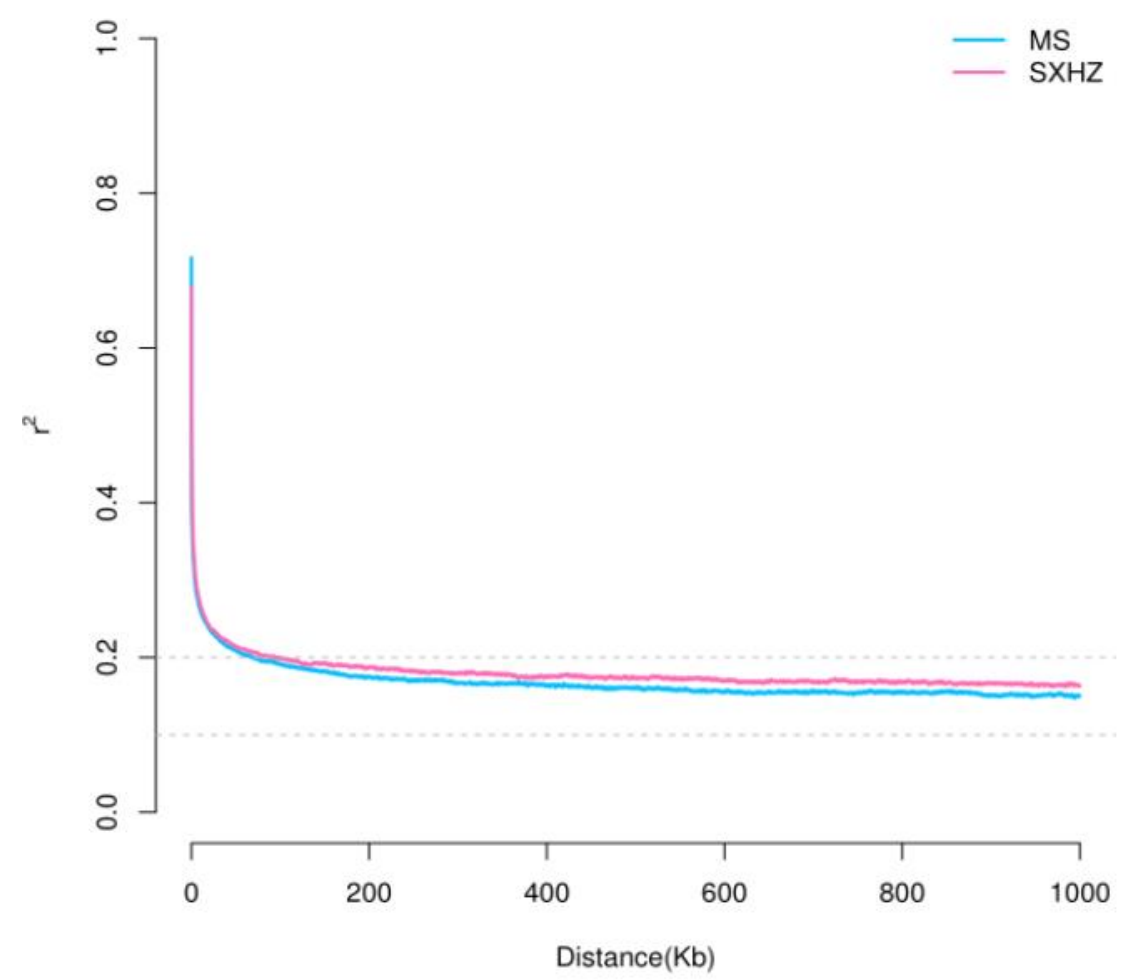

Note:The red line is the LD attenuation curve of Shenxian pigs, and the blue line is the LD attenuation curve of big white pigs.

Figure 8 LD attenuation diagram of the Shenxian pig and the Meishan pig populations

\subsection{Selection signal analysis results}

\subsubsection{Fst and $\theta \pi$ analyses}

In order to study the selection preferences of the Shenxian pigs, this study analyzed the selection signals of both the large white pigs and the Shenxian pigs. Ten Shenxian pigs forming a population, and nine large white pigs forming a population, were selected for this study's signal analysis process. The detection strategy of combining population differentiation index Fst and nucleic acid diversity $\theta \pi$ was adopted. A population differentiation index (Fst) method was used to anchor the regions of the genome differentiation between the populations. A nucleic acid diversity $(\theta \pi)$ method was used to anchor the regions of the reduced polymorphism within the populations. The regions identified by the two methods were selected as the candidate regions for the purpose of improving the reliability of the results. In addition, in order to reduce the impacts of any outliers, this study utilized a sliding window detection method. The sliding window size was set as $10 \mathrm{kbp}$ and the sliding window step was set as $1 \mathrm{kbp}$. In order to compare the results of the two analyses, the Fst values and the $\theta \pi$ ratio $(\theta \pi$ large white pig/ $\theta \pi$ Shenxian pig) values were adjusted to a unified scale using a minimum/maximum 
conversion process, as detailed in Figure 9.

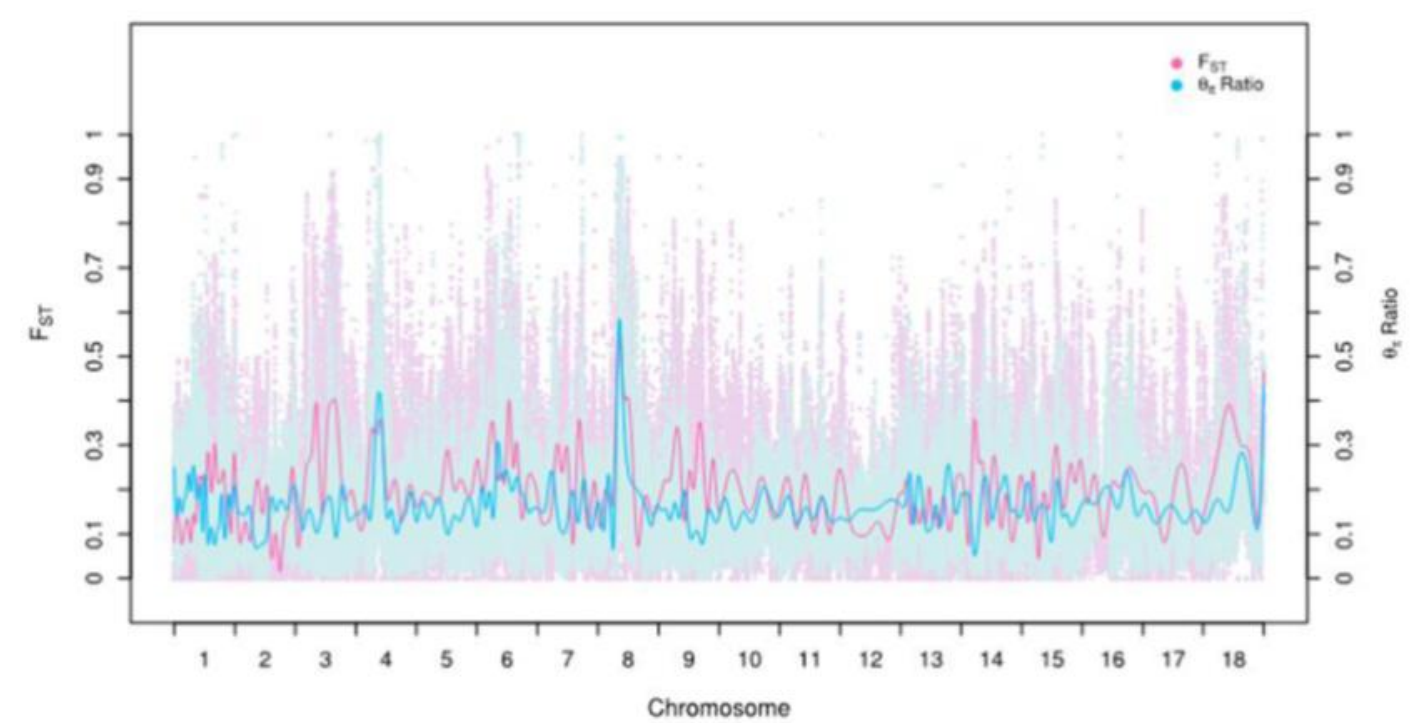

Note: Figure 9 shows the results of Fst and $\boldsymbol{\theta} \pi$ following the completions of size conversions; Red represents the results of the Fst and blue represents the results of $\theta \pi$ ratio.

\section{Figure 9 Fst and $\theta \pi$ Manhattan Plot}

\subsubsection{Inference of the selected sites}

The top $1 \%$ window of the Fst slide window analysis and the top $1 \%$ window of the $\theta \pi$ analysis were screened in order to obtain 1,509 selected sites, and the selected sites were then annotated. Figure 10 and Figure 11, respectively, show the distributions of the selected variation sites in the chromosome and genome. Figure 10 shows that when compared with large white pigs, the Shenxian pigs had displayed more selection variations on chromosome 8 and less variations on the other chromosomes. As can be seen from Figure 11, the selection of those sites was mainly concentrated between the intergenic region and the intron, while the selection in other regions was less. However, the variations of the intergenic regions and intron had little effect on the gene expressions, which may be due to the fact that the changes in selection pressure after species differentiation had resulted in major differences between intron and intergenic regions. 


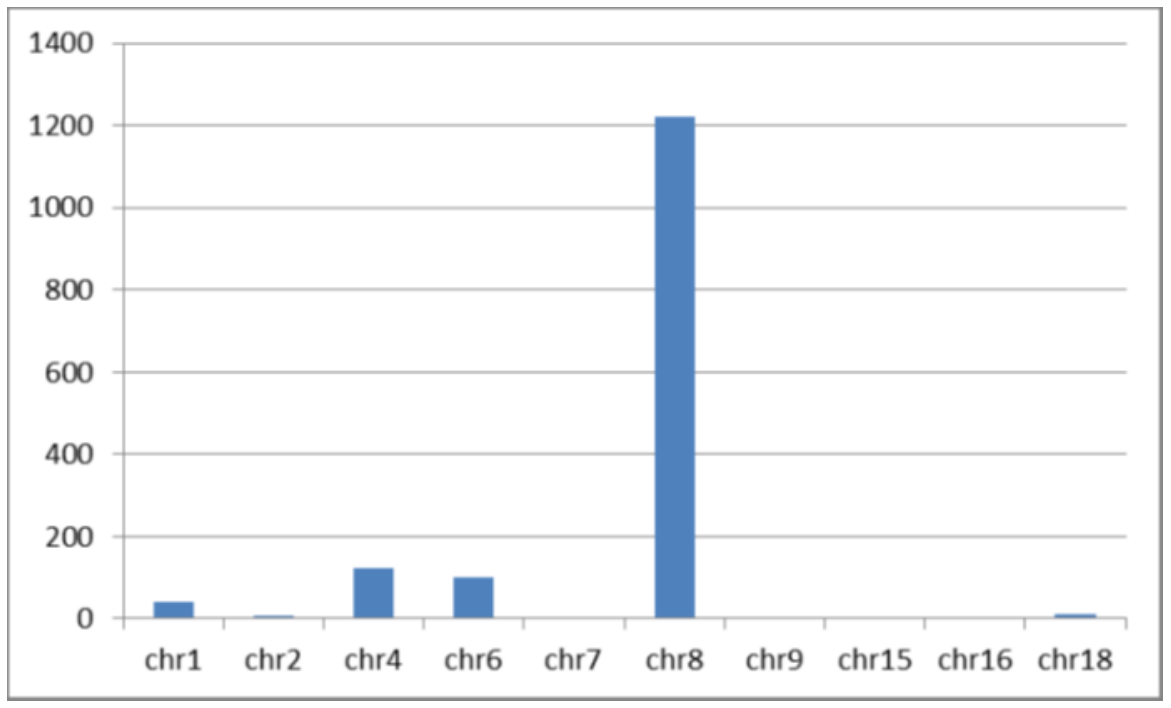

Note: In the figure, the abscissa represents the chromosome number; and the ordinate represents the number of selected sites.

Figure 10 Distribution of the variations on the chromosomes

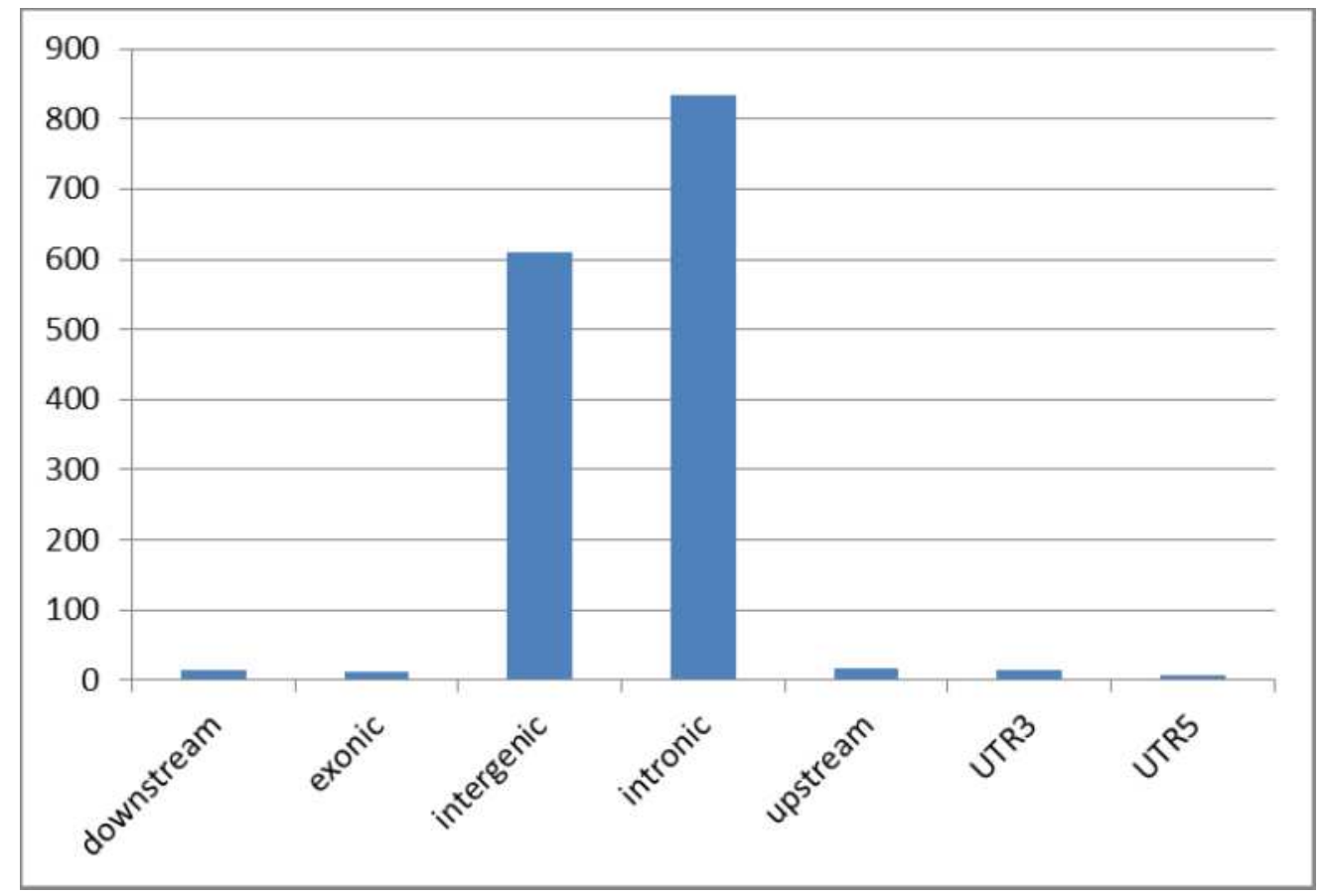

Note: In the figure, the abscissa represents the gene region; and the ordinate represents the number of selected sites.

Figure 11 Distribution of the variation locations

\subsubsection{Functional enrichment analysis of the candidate gene $G O$}

In the current research investigation, the 19 differentially expressed genes at 1,509 sites obtained through the screening process were subjected to functional enrichment and 
significance testing processes. The GO enrichment consisted of a total of three parts: Molecular function (MF); biological process (BP); and cell composition (CC), which were enriched to 421 entries. Then, through the enrichment analyses of the genes, the biological processes involved in those genes were successfully identified. From the result, the selection regions of the Shenxian pigs relative to the large white pigs during the processes of genetic evolution and domestication could be identified, and the selection characteristics of the Shenxian pig population were more clearly understood.

As shown in Figure 12, the main involved biological processes included lactation; body fluid secretion; host interaction; regulatory pathways of the $G$ protein coupled receptor signal transduction; dentinogenesis; neuropeptide signaling pathway; breast development; chemical synaptic transmission; antegrade synaptic signaling, and so on.

In addition, as can be seen in Figure 13, the cell compositions indicated that the candidate genes may also participate in extracellular space; synapse; ciliated body; protoplasm membrane composition; plasma membrane composition; postsynaptic membrane; plasma membrane interval; basal outer plasma membrane; and synaptic membrane processes.

As detailed in Figure 14, the molecular function enrichment results revealed that the candidate genes were involved in inorganic anion transmembrane transporter activities; anion transmembrane transporter activities; hydroxyl acid binding; organic acid binding; fatty acid binding; excitatory cell ligand ion channel activities; neurotransmitter binding; insulin-like growth factor binding; chloride ion binding; cell apoptosis Ligand ion channel activities, and other molecular functions.

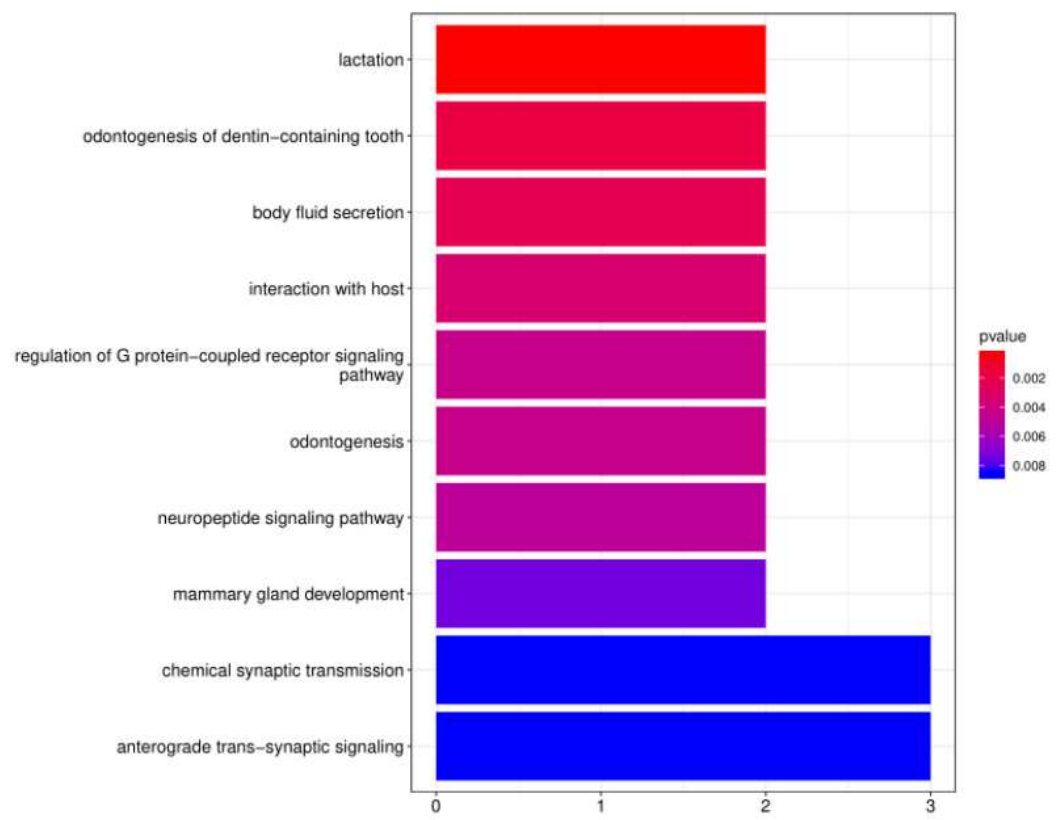

Note: In the figure, the abscissa represents the number of enriched genes; and the ordinate represents the biological processes of enrichment.

Figure 12 Enrichment analysis results of the gene biological processes 


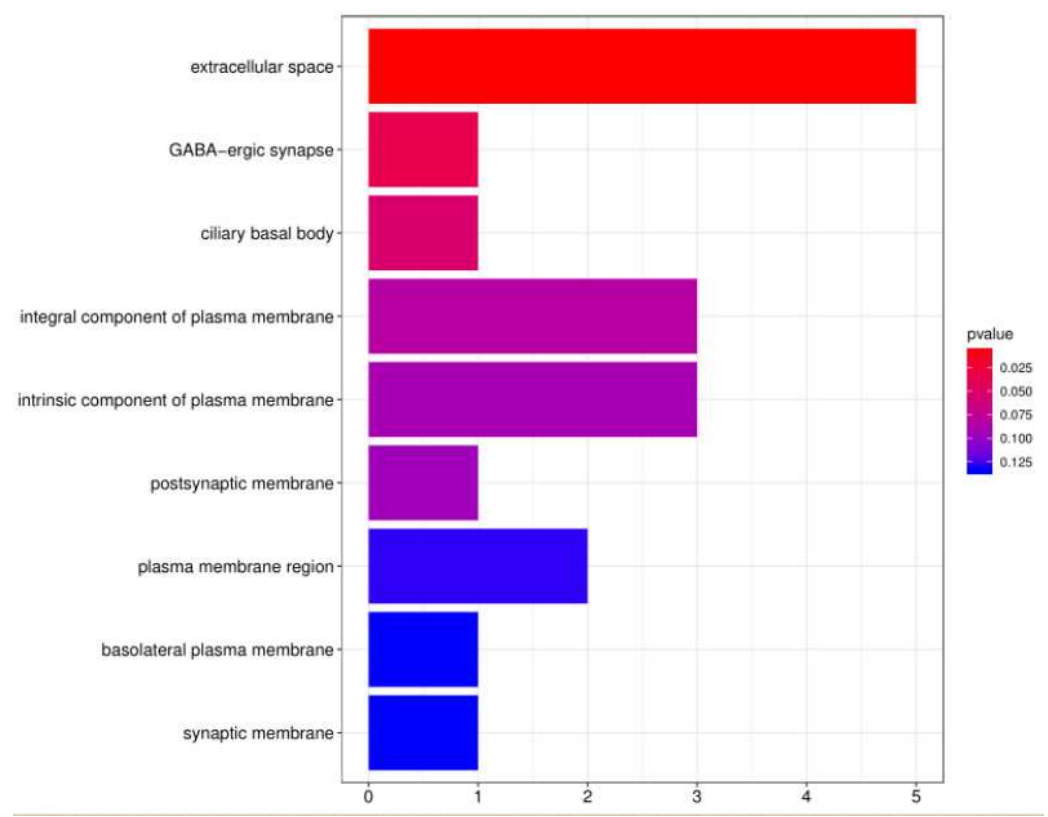

Note: In the figure, the abscissa represents the number of enriched genes; and the ordinate represents the cell compositions.

Figure 13 Enrichment analysis results of the gene cell compositions

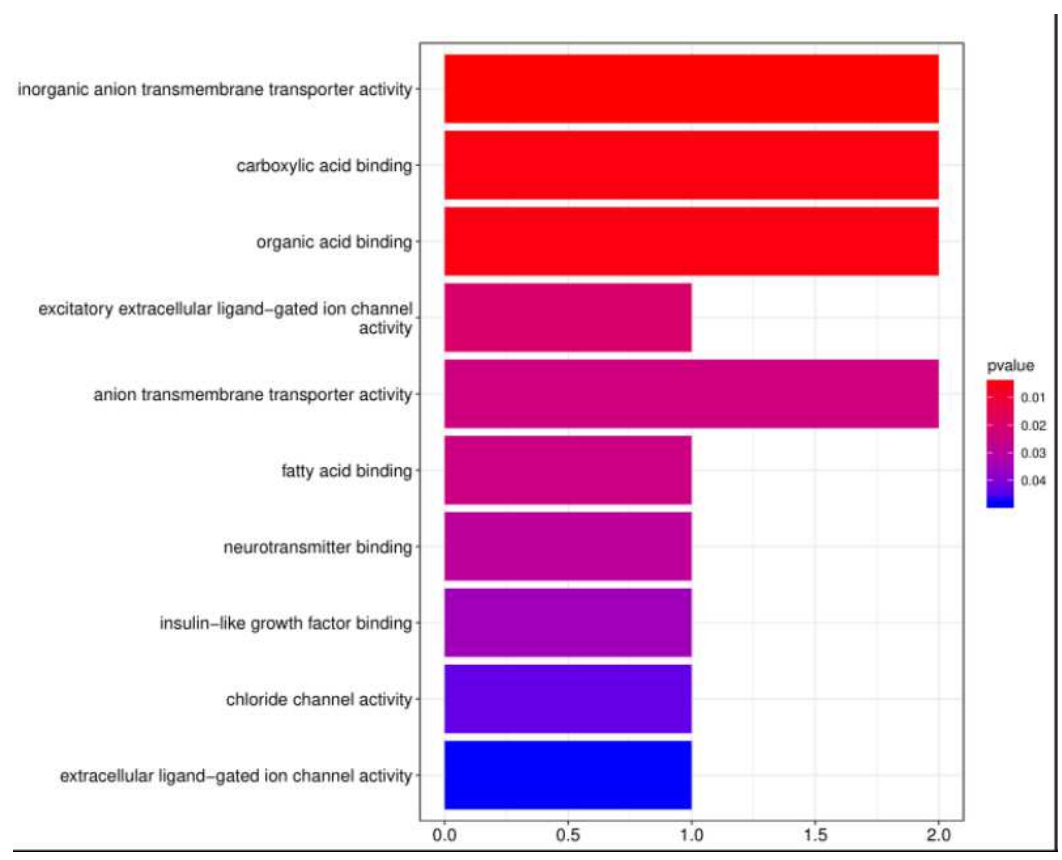

Note: In the figure, the abscissa represents the number of enriched genes; and the ordinate represents the molecular enriched functions.

Figure 14 Enrichment analysis results of the gene molecular functions

\subsubsection{Genes significantly enriched into pathways}

Through the KEGG pathway analyses of the candidate genes revealed that those genes were mainly involved in two pathways, as detailed in Figure 15. It was found that 
among the 19 genes, six genes were involved in the neuroactive receptor interaction pathways, and three genes were involved in phospholipase D signaling pathways.

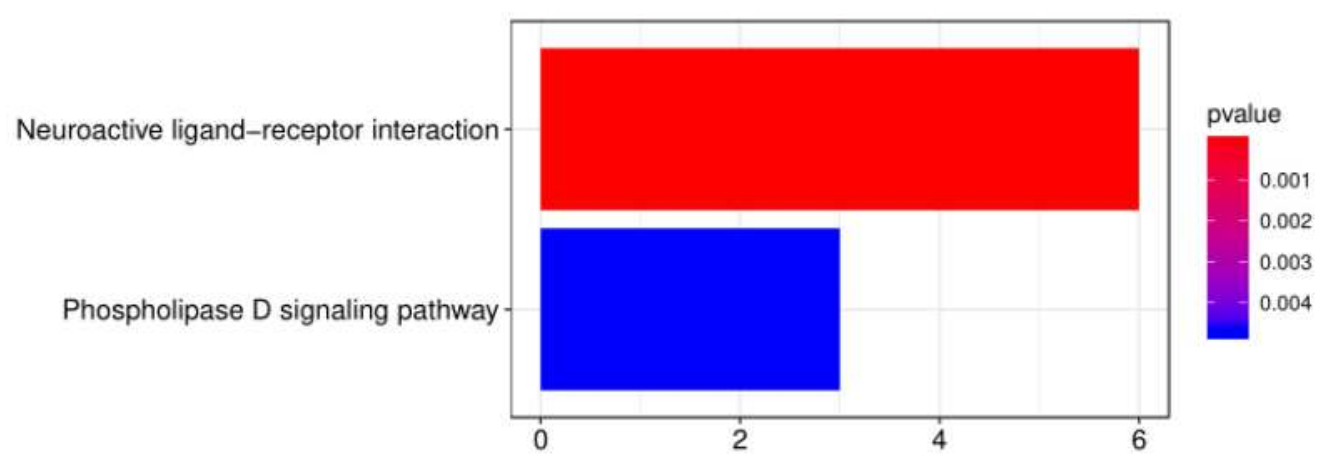

Note: In the figure, the enriched KEGG pathway terms include the horizontal axis representing the number of genes, and the vertical axis representing the enriched pathways.

Figure 15 KEGG pathway analysis results

In this experimental study, the selection regions of the local variety (Shenxian pig) and western commercial variety (large white pig) were analyzed. The results indicated that in some of the signaling pathways, the two varieties had the same artificial selections. However, the majority of the selection regions were different. The differences between the natural selections and artificial selections may have caused the results in the two pig varieties. For example, the large white pig variety has developed into a mature commercial variety, and has been widely used throughout the world. Meanwhile, in the case of the Shenxian pig variety, this breed has been essentially developed as a local variety. Therefore, further research will be required in order to retain its individual characteristics and excellent genes. This study only focused on the variety selection signals of the Shenxian and large white pigs, and determined the selection characteristics of the introduced Shenxian pig. However, the genetic characteristics of the Shenxian pig breed as a local variety in China remain unclear. In the future, complete selection signal analyses of Shenxian pigs and different other pig varieties will be conducted. Also, selection signal analyses of wild populations will also be carried out in order to deepen our understanding of the artificial selection traits of Shenxian pigs. However, the selection characteristics of the examined different breeds were better understood through the selection signal analyses of other local varieties. In addition, the functional genes were mined to truly understand the variety diversity and population characteristics of Shenxian pigs.

\section{Discussion}

In this study, the screened genes included CXCL8, GLRB, CD180, TMOD2, AFP, ALB, RASSF6, AMBN, CSN2, CSN3, DMTF1, IGFBP7, IMPAD1, NAP114, NPY1R, 
ODAM, SLC4A4, WDR5, and AFM. Among those genes, CXCL8 ${ }^{[4,5]}$, CD180 ${ }^{[6]}$, AFP ${ }^{[7]}$, RASSF6 ${ }^{[8]}$, and IGFBP7 have been proved to be related to immune diseases, and these genes are closely related to human diseases. For example, CXCL8 is a chemokine which is expressed in a variety of tissues. In human studies, it has been found that the CXCL8 gene is related to cancer, and its expression levels in cancer tissue are higher than those in other tissue ${ }^{[9]}$. At the same time, it may also cause hypertension ${ }^{[10]}$, and its expression levels have been found to be higher in patients with chronic hepatitis B ${ }^{[11]}$. Furthermore, this gene can cause inflammatory reactions in pigs ${ }^{[12]}$. It is of major significance to study this gene for disease research purposes. In previous studies of diseases, CD180 was found to be related to systemic lupus erythematosus. The expression levels of CD180 in the cells of patients with systemic lupus erythematosus were higher than those of patients without systemic lupus erythematosus. At the same time, CD180 is considered to be related to chronic lymphoproliferative diseases ${ }^{[13]}$. However, this gene has not been reported in previous studies regarding pigs. The AFP gene is related to human liver cancer, lung cancer, pancreas, and other diseases, and the research regarding AFP in pigs is also rare ${ }^{[14]}$. The RASSF6 gene is known to be associated with colon cancer disease, and can promote the growth of colon cancer cells ${ }^{[15]}$. The IGFBP7 gene ${ }^{[16,17]}$ has been determined to be related to osteoarthritis. The gene expression levels in cartilage tissue of patients with osteoarthritis have been found to be high, and the gene can accelerate chondrocyte apoptosis by inhibiting chondrocyte proliferation. The DMTF1 ${ }^{[18,19]}$, WDR5, and other genes and known to be related to cell proliferation. The DMTF1 gene is also related to cancer diseases ${ }^{[20]}$. The WDR5 gene plays a certain role in tissue regeneration and bone tissue development ${ }^{[21]}$, and this gene and family protein have been confirmed to be able to maintain the normal development of pig preimplantation embryos ${ }^{[22 \text { to } 24]}$. The CSN2 ${ }^{[25,26]}$ and CSN3 ${ }^{[27]}$ genes have been found to be related to lactation. At the same time, there were also other genes observed in the selected regions. For example, the AMBN gene may be related

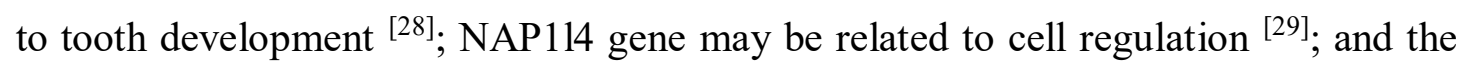
IMPAD1 gene may be related to bone development ${ }^{[30]}$. However, there have been few studies conducted regarding the aforementioned genes in pig breeds.

\section{Conclusions}

In the present research investigation, through the analyses of the genetic relationships of the Shenxian pig variety and the large white and Meishan pig varieties, it was determined showed that the Shenxian pigs had higher genetic diversity than the large white pigs, but their genetic richness of population was lower than that of the Meishan pigs. In addition, the degree of domestication in the Shenxian pig population was 
relatively low, and traces of artificial selections were not obvious. The screened and selected genes were annotated, and 19 genes were found to be enriched in the biological entries. Among those genes, CXCL8, CD180, RASSF6, AFP, and IGFBP7 were proven to be related to immune diseases; DMTF1 and WDR5 were found to be related to cell proliferation; and CSN2 and CSN3 were confirmed to be related to lactation. This study determined that the Shenxian pig variety had displayed no obvious selections in reproduction, growth, meat quality traits, and so on. Therefore, the next step will be to strengthen the selection of those traits.

\section{Experimental materials and methods}

\subsection{Experimental design}

Analyze the population genetic structure of Shenxian pigs and Large White pigs, analyze the genetic relationship between the two, perform selection signal analysis, screen the selected regions of Shenxian pigs relative to Large White pigs, annotate the candidate genes of the region, and analyze differential expression To explore the selection preference of Shenxian pigs in the evolutionary process.

\subsection{Sample size}

The t-test method of the paired test is to deduce the number of animals required for the test. When a significant level of $\alpha=0.05$ is to be reached, the content of the sample can be determined by the following formula:

$$
\mathrm{n}=\left(\mathrm{t}_{0.05} * \mathrm{~S}_{\mathrm{d}}\right)^{2} / \mathrm{d}^{2}
$$

Among them, $\mathrm{S}_{\mathrm{d}}$ is the variance of the difference between the pairs obtained by previous experiments or experience; $d$ is the mean difference when the expected difference is significant.

\subsection{Experimental materials}

The Shenxian pigs used in this experiment were selected from Hebei Zhengnong Animal Husbandry Co., Ltd., from the resource group of 150 sows from 10 families of Shenxian pigs, 10 21-day-old healthy and newly weaned piglets were selected from each family. Pick one of each and use ear tag tongs to collect the ear tissues of pigs in Shenxian County. Before collection, cut the ear tissues and sterilize them with $75 \backslash \%$ alcohol. Use ear tag tongs to punch and sample the pig ears. Take about 100mg samples. The $95 \backslash \%$ alcohol EP tube is transported back to the laboratory in an ice bag and stored in a refrigerator at $-20{ }^{\circ} \mathrm{C}$ for later use. During sampling, transportation and storage, ensure that the DNA sample is not contaminated and used for subsequent genomic DNA extraction. 


\subsection{Whole genome resequencing}

The 10 DNA samples of Shenxian pigs that passed the inspection were handed over to Beijing Compson Agricultural Technology Co., Ltd. to construct a gene library and complete the whole genome resequencing work. At present, the second-generation sequencing technology is widely used ${ }^{[31,32]}$, Based on the Illumina technology sequencing platform, the paired-end sequencing (Paired-END) method is used, and the sequencing strategy uses Illumina PE150 to complete the whole genome resequencing of 10 DNA samples, ensuring that each sample produces no less than $28 \mathrm{~Gb}$ raw data. In the current study, the two subpopulations of Shenxian pigs were re-sequenced for the purpose of obtaining the genomic information. A large number of high accuracy SNPs, InDel, and other variation information were obtained by comparing the results with the reference genome. Subsequently, the variation information was successfully detected, annotated, and counted.

\subsection{Data processing}

This test sample uses the whole genome resequencing data (sequencing depth 10X) of 10 Shenxian pigs ( 5 cucumber-mouthed pigs and 5Wuhuatou pigs). The output of the genome data comparison is the sam file. Since the sorting method of the sam file cannot be used for subsequent analysis, the Samtools software ${ }^{[33]}$ is used to convert the sam file into a file in chromosome sorting format, that is, the bam format. Chromosome rearrangement is to arrange the entries of the same chromosome in the file in ascending order, and merge the two files of the same sample.Download the resequencing genome data of 9 large white pigs and 10 Meishan pigs from the SRA database of the NCBI database (http://www.ncbi.nlm.nih.gov/sra). The downloaded file is generally in sra format and needs to be used The SRA Toolkit tool converts the downloaded sequencing data into a fastq format file for SNP identification.

\subsection{SNP detection and annotation}

Use GATK and VarScan software ${ }^{[34]}$ to perform SNP detection at the same time, so as to ensure that the obtained SNP site information will not be affected by the deviation of base misalignment caused by InDel mutation. GATK detection SNP code: java -jar GenomeAnalysisTK.jar glm SNP -R ref.fa -T UnifiedGenotyper -I test.sorted.repeatmark.bam -o test.raw.vcf. In order to ensure the accuracy of SNP information, strict testing conditions are set when detecting SNP site information: $<1>$ The minimum number of end reads greater or equal to $4 ;<2>$ The minimum quality value Q20 greater or equal to $90 ;<3>$ Minimum coverage greater or equal to 6 ; $<4>$ P value Less than or equal to 0.01 . InDel detection and annotation: The same use of GATK and VarScan software for InDel detection can eliminate errors caused by 
SNP variation. GATK detection InDel code: java -jar GenomeAnalysisTK.jar glm InDel -R ref.fa -T UnifiedGenotyper -I test.sorted.repeatmark.bam -o test.raw.vcf. The detection conditions are consistent with SNP. The detected SNP and InDel mutation sites also need quality control, remove untrusted data sites, correct the quality value, and output the file in VCF format after correction. Use R language ${ }^{[35,36]}$ and ANNOVAR software ${ }^{[37]}$ to perform mutation information Sort and comment.

\subsection{Population genetic structure analysis based on SNP}

This study performed principal component analysis (PCA) on the Shenxian, large white, and Meishan pig populations using Plink (V1.90) software. The top two eigenvector values were selected for analysis purposes. Generally speaking, the smaller the spatial straight-line distance in the PCA map was, the closer the individual genetic relationship would be. The genetic distance matrix of IBS was constructed using Plink (V1.9) ${ }^{[38]}$, and then a phylogenetic tree was constructed using a neighbor joining $(\mathrm{NJ})$ method with the genetic distance matrix. A genetic evolutionary tree was drawn using mega software. Linkage disequilibrium analyses were carried out using PopLDdecay software, for the purpose of analyzing the associations of the alleles among the three populations. In addition, the genetic richness of the populations were determined according to the attenuation rates of the LD. Admixture software was adopted for this study's population genetic structure analyses, which was helpful in furthering the understanding of the evolution processes and identifying the migrating individuals and heterozygotes. As a result, the subgroup to which an individual belonged could be accurately determined by association studies of its genotype and phenotype.

\section{Declarations}

\subsection{Ethics approval and consent to participate}

We obtained written informed consent to use the animals in this study from Hebei Zhengnong Animal Husbandry Co., Ltd of the shenxian pigs respectively. The care and treatment of the shenxian pigs comply with the guidelines of animal use protocols approved by the Animal Care and Ethics Committee of the Hebei Agricultural University.

\subsection{Consent for publication}

Not applicable.

\subsection{Availability of data and materials}

The data sets supporting the results of this article are included in this manuscript and its additional information files. Download the resequencing genome data of 9 large 
white pigs and 10 Meishan pigs from the SRA database of the NCBI database (http://www.ncbi.nlm.nih.gov/sra).

\subsection{Competing interests}

The authors declare that they have no competing interests.

\subsection{Funding}

This work was supported by the Hebei Province Pig Industry Technology Innovation System Project (HBCT2018110203). The funding bodies played no role in the design of the study and collection, analysis, and interpretation of data and in writing the manuscript.

\subsection{Authors' contributions}

Diao Liu wrote the main manuscript text and prepared figures and tables.All authors reviewed the manuscript.

\subsection{Acknowledgements}

We thank the Hebei Province Pig Industry Technology Innovation System Project for funding, we thank Hebei Zhengnong Animal Husbandry Co., Ltd. for the research samples provided, thank every member of our office for their hard work, and we also thank the staff for helping us collect samples.

\subsection{Authors' information}

${ }^{1}$ College of Animal Science and Technology, Hebei Agricultural University, Baoding 071000, Hebei. ${ }^{2}$ Hebei Veterinary Drug Supervision Institute, Shijiazhuang 050051, Hebei. ${ }^{3}$ Fishery Technology Extension Station, Wanquan District, Zhangjiakou, 076250, Hebei. ${ }^{4}$ Hebei Zhengnong Animal Husbandry Co., Ltd., Xinji 052360, Hebei.

\section{References}

1. Giuffra,E.,Kijas,J.M.,Amarger,V.,et al. The origin of the domestic pig: independent domestication and subsequent introgression[J]. Genetics. 2000;154(4):1785-1791.

2. Groenen,M.A.,Archibald,A.L.,Uenishi,H.,et al.Analyses of pig genomes provide insight into porcine demography and evolution[J]. Nature. 2012;491(7424):393398.

3. Ai,H.,Fang,X., Yang,B.,et al.Adaptation and possible ancient interspecies introgression in pigs identified by whole-genome sequencing.NatGenet.2015; 47(3):217-225.

4. Wang,X., Li,Y., Li,L., et al. Porcine CXCR1/2 antagonist CXCL8(3-72)G31P 
inhibits lung inflammation in LPS-challenged mice. Sci Rep. 2020; 10(1):1210.

5. Ukaszewicz-Zajc,M., Pczek,S., Mroczko,P.,et al., 2020 The significance of cxcl1 and cxc18 as well as their specific receptors in colorectal cancer. Cancer Management and Research.12, 8435-8443.

6. Dong,G., Yao,X., Yan,F., et al., 2018 Ligation of cd180 contributes to endotoxic shock by regulating the accumulation and immunosuppressive activity of myeloidderived suppressor cells through stat3. Biochimica et Biophysica Acta (BBA) Molecular Basis of Disease .1865(3):535-546.

7. B,Q.C.A., A,Y.Z., A,W.C., et al., 2020 Apios americana medik flowers polysaccharide (afp) alleviate cyclophosphamide-induced immunosuppression in icr mice. International Journal of Biological Macromolecules. 144, 829-836.

8. Vogel,N., Piras-Straub,K., Busch,M., et al.,2020 Influence of pml, rassf6 and nlrp12 on growth and recurrence of human hepatocellular carcinoma. Journal of Hepatology. 73, S286.

9. Tongbo Yi, Xiaoqing Zhou, Kai Sang, et al.,2019 Activation of Incrna lnc-slc4a11 induced by $\mathrm{h} 3 \mathrm{k} 27$ acetylation promotes the development of breast cancer via activating cxcl8 and nf-kb pathway. Artificial Cells. 47(1),3765-3773.

10. Young,H., Kim, Won,D., et al., 2018 Sulfatase 2 mediates, partially, the expression of endothelin-1 and the additive effect of ang ii-induced endothelin-1 expression by cxcl8 in vascular smooth muscle cells from spontaneously hypertensive rats. CYTOKINE.

11. Sharif,G.M., Wellstein,A. 2015 Cell density regulates cancer metastasis via the hippo pathway. Future Oncology. 11(24), 3253-3260.

12. Hu,Y.H., Chen,L., Sun,L. 2011 Cxcl8 of scophthalmus maximus: expression, biological activity and immunoregulatory effect. Developmental and Comparative Immunology. 35(10),1032-1039.

13. Jing,Yan, Sheng, et al.,2018 characteristics of cd180 expression and its diagnostic value in b cell chronic lymphoproliferative disorders. Zhongguo shi yan xue ye xue za zhi. 26(6), 1811-1815.

14. Kim,J.G., Nonneman,D., Vallet,J.L., et al., 2003 Mapping of the porcine alphafetoprotein (afp) gene to swine chromosome 8. Animal Genetics. 33(6), 471-472.

15. Wang,H., Yan,B., Zhang,P., et al., 2020 Mir-496 promotes migration and epithelial-mesenchymal transition by targeting rassf6 in colorectal cancer. Journal of Cellular Physiology(1). 
16. Zhang,L., Lian,R., Zhao,J., et al., 2019 Igfbp7 inhibits cell proliferation by suppressing akt activity and cell cycle progression in thyroid carcinoma. Cell and Bioscience. 9(1), 44.

17. Chenyi Ye, Weiduo Hou, Mo Chen, et al.,2020 IGFBP7 acts as a negative regulator of RANKL-induced osteoclastogenesis and oestrogen deficiencyinduced bone loss. Cell Proliferation.53(2).

18. Tschan,M.P., Federzoni,E.A., Haimovici,A., et al. 2015 Human dmtflbeta antagonizes dmtflalpha regulation of the p14(arf) tumor suppressor and promotes cellular proliferation. BBA - Gene Regulatory Mechanisms.1849(9),1198-1208.

19. Xueliang Yang, Lou, Minghua Wang, et al., 2018 Mir-675 promotes colorectal cancer cell growth dependent on tumor suppressor dmtfl. Molecular Medicine Reports.19(3):1481-1490.

20. Peng,Y., Dong,W., Lin,T.X.,et al., 2015 Microrna-155 promotes bladder cancer growth by repressing the tumor suppressor dmtfl. Oncotarget. 6(18),16043-16058.

21. B,R.L.A., C,Z.L., B,E.S.,et al., 2020 Lncrna hottip enhances human osteogenic bmscs differentiation via interaction with wdr5 and activation of wnt/beta-catenin signalling pathway. Biochemical and Biophysical Research Communications. 524( 4), 1037-1043.

22. Gallenberger,M., Meinel,D.M., Kroeber,M., et al. 2011 Lack of wdr36 leads to preimplantation embryonic lethality in mice and delays the formation of small subunit ribosomal rna in human cells in vitro. Archiv Der Mathematik. 6(2), 121 127.

23. Maserati,M., Walentuk,M., Dai,X.,et al., 2011 Wdr74 is required for blastocyst formation in the mouse. PLoS ONE. 6(7), e22516-.

24. Ye,B., Zhuo,L., Ying,W.,et al.,2011 Wdr82, a key epigenetics-related factor, plays a crucial role in normal early embryonic development in mice1. Biology of Reproduction.84(4):756-64.

25. Suteu,M., Vlaic,A., Drban,S.V. 2019 Characterization of a novel porcine csn2 polymorphism and its distribution in five european breeds. Animals : an Open Access Journal from MDPI. 9(7).

26. Kumar,S., Singh,R.V., Kumar,A., et al., 2019 Analysis of beta-casein gene (csn2) polymorphism in tharparkar and frieswal cattle. Indian Journal of Animal Research(of).

27. Curi, Rogério,A., De,H.N., et al., 2005 Effects of csn3 and $\operatorname{lgb}$ gene 
polymorphisms on production traits in beef cattle. Genetics and Molecular Biology.

28. Ting,L., Meiyi,L., Xiangmin,X.,et al., 2018 Whole exome sequencing identifies an ambn missense mutation causing severe autosomal-dominant amelogenesis imperfecta and dentin disorders. International Journal of Oral Science. 10(4):223231.

29. Tanaka,T., Hozumi,Y., Martelli,A.M., et al., 2019 Nucleosome assembly proteins nap111 and nap114 modulate p53 acetylation to regulate cell fate. Biochimica et Biophysica Acta (BBA) - Molecular Cell Research. 1866(12):118560-.

30. Vissers,L.L.M., Lausch,E., Unger,S., et al., 2011 Chondrodysplasia and abnormal joint development associated with mutations in impad1, encoding the golgiresident nucleotide phosphatase, gpapp. AMERICAN JOURNAL OF HUMAN GENETICS. 88(5).

31. Levy,S.E., Myers,R.M.,2016 Advancements in next-generation sequencing. Annu Rev Genomics Hum Genet 17(1), 95-115.

32. Slatko Barton,E., Gardner Andrew,F., Ausubel Frederick,M.,2018 Overview of Next-Generation Sequencing Technologies.122(1):59.

33. Mardis,E.R.,2008 The impact of next-generation sequencing technology on genetics.Trends Genet 24:133-141.

34. Shan Gao, Jianhong Ou, Kai Xiao.,2014b R language and Bioconductor bioinformatics application. Tianjin: Tianjin Science and Technology Translation Publishing Company, 2014.

35. Kalyan Sudhaka.,2018b Python vs. R Programming Language. 8(8):70-79.

36. Kai,W.,Mingyao,L.,Hakon,H.,2014 ANNOVAR:functional annotation of genetic variants from high-throughput sequencing data[J].Nucleic Acid Research.16(38):e164.

37. Group,T.I.S.M.W.,2001 A map of human genome sequence variation containing 1.42 million single nucleotide polymorphism. Nature. 15 .

38. Purcell,S., Neale,B.,Todd-Brown,K.,et al.,2007 PLINK: a tool set for wholegenome association and population-based linkage analyses[J].Am J Hum Genet81(3): 559-575. 
Figures
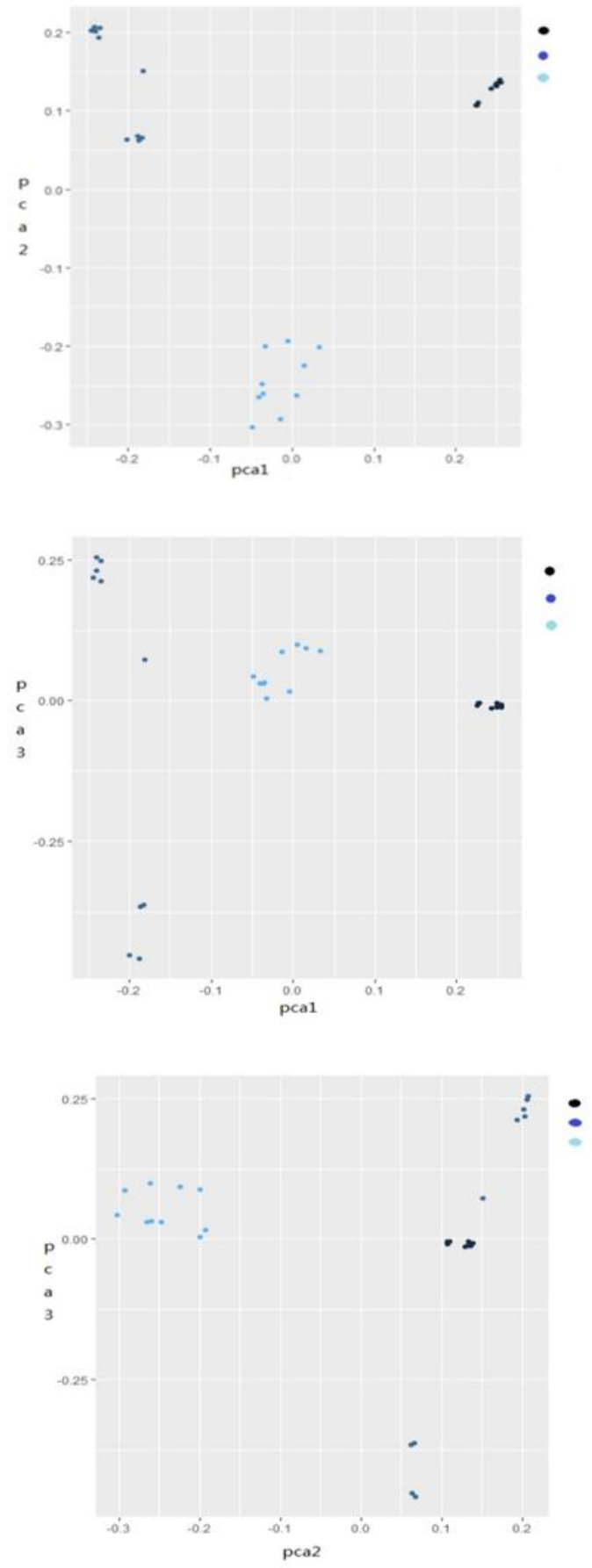

\section{Figure 1}

Note: Black represents big white pigs, dark blue represents Meishan pigs, light blue represents Shenxian pigs. Figure 1 to Figure 3 Principal component analysis results 


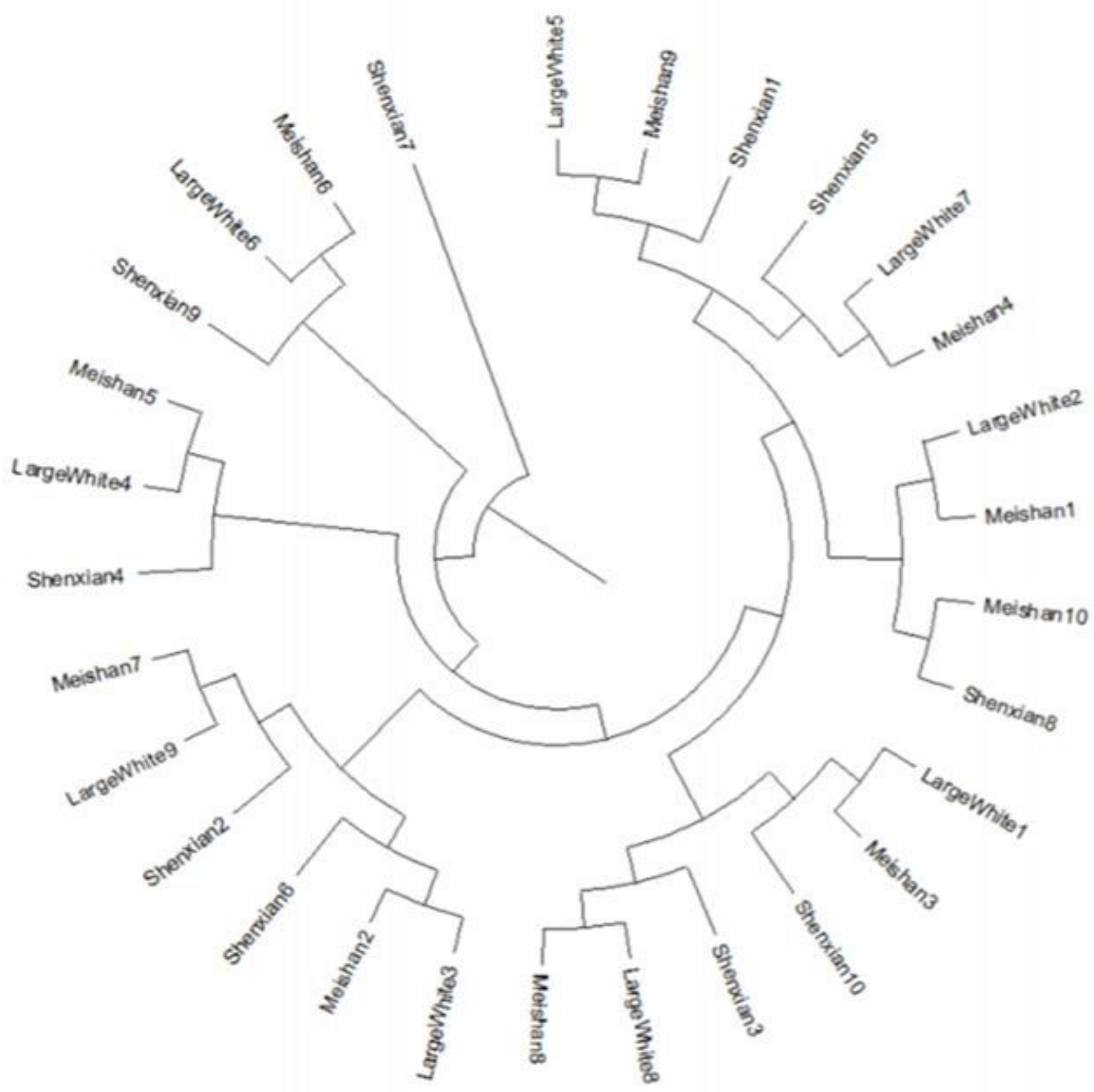

Figure 2

2 Phylogenetic tree 


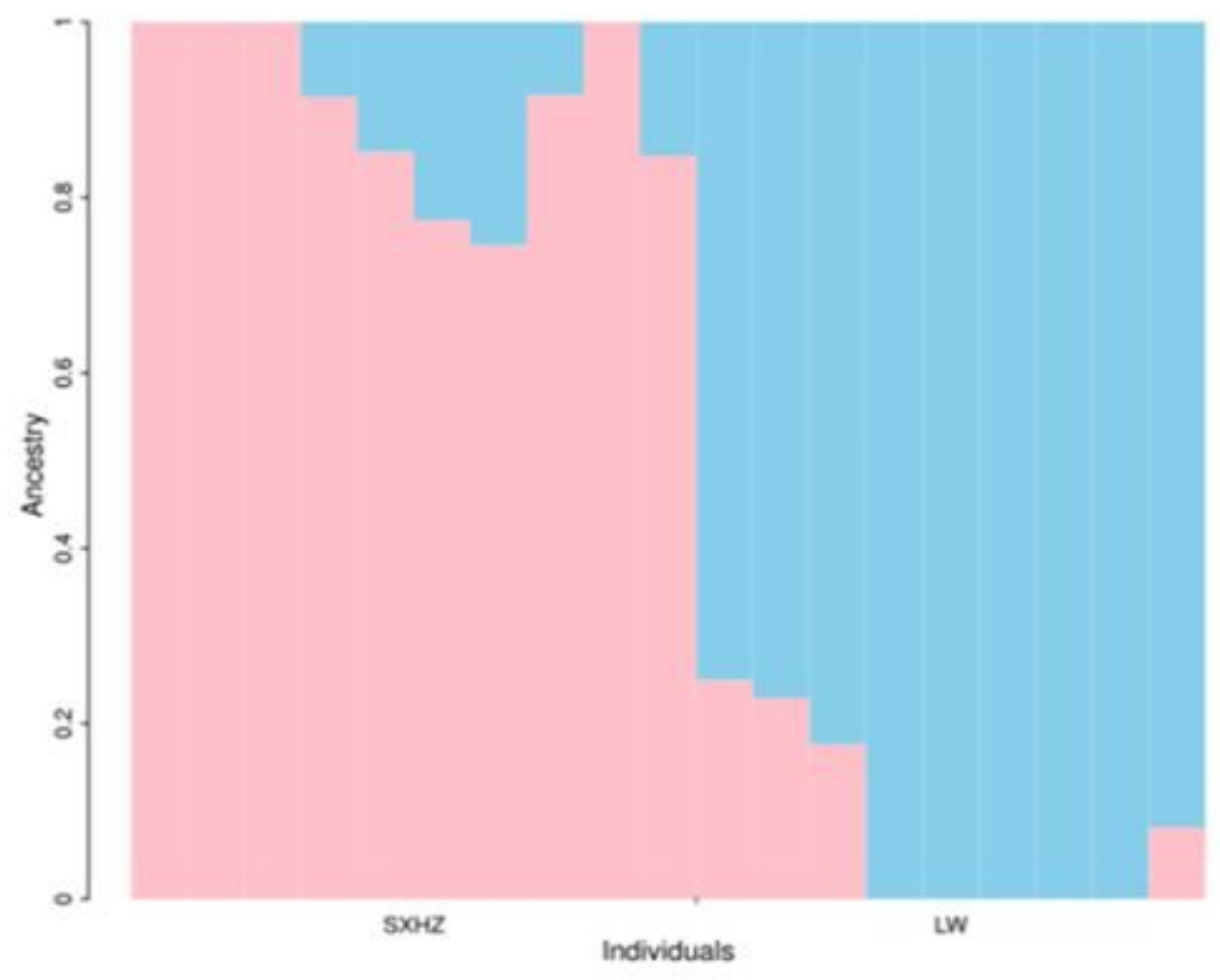

\section{Figure 3}

Note: The picture shows the group structure of Shenxian pigs and large white pigs. Pink means the Shenxian pigs , blue means the large white pigs. The abscissa represents the individuals in the group, and the ordinate represents the included ancestry. Population structures of the Shenxian and large white pig breeds 


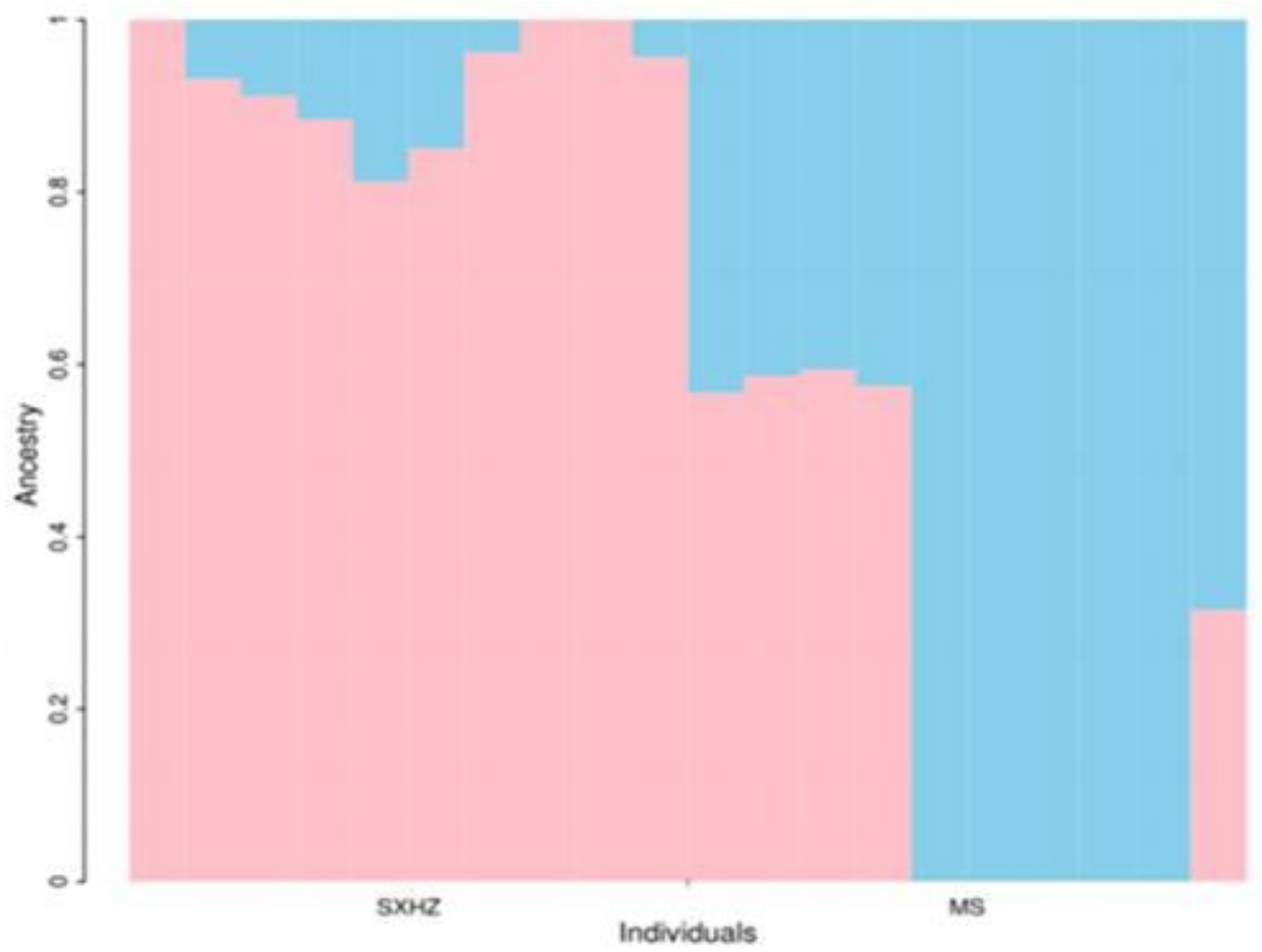

\section{Figure 4}

Note: The picture shows the group structure of Shenxian pigs and Meishan pigs. Pink means the Shenxian pigs, blue means the Meishan pigs in. The abscissa represents the individuals in the group, and the ordinate represents the included ancestry. Population structures of the Shenxian and Meishan pig breeds 


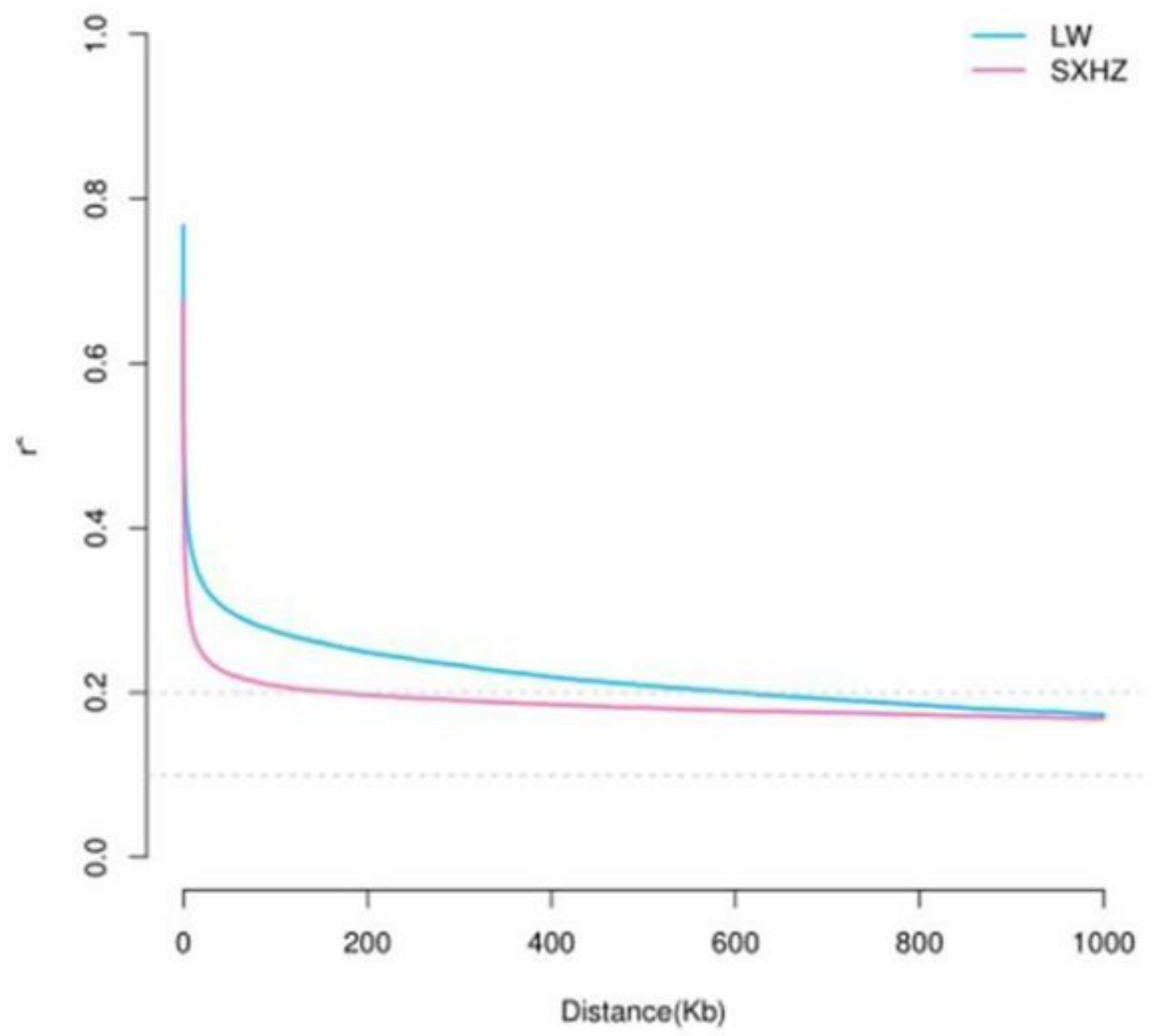

Figure 5

Note:The red line is the LD attenuation curve of Shenxian pigs, and the blue line is the LD attenuation curve of big white pigs. LD attenuation diagram of the Shenxian pig and the large white pig populations 


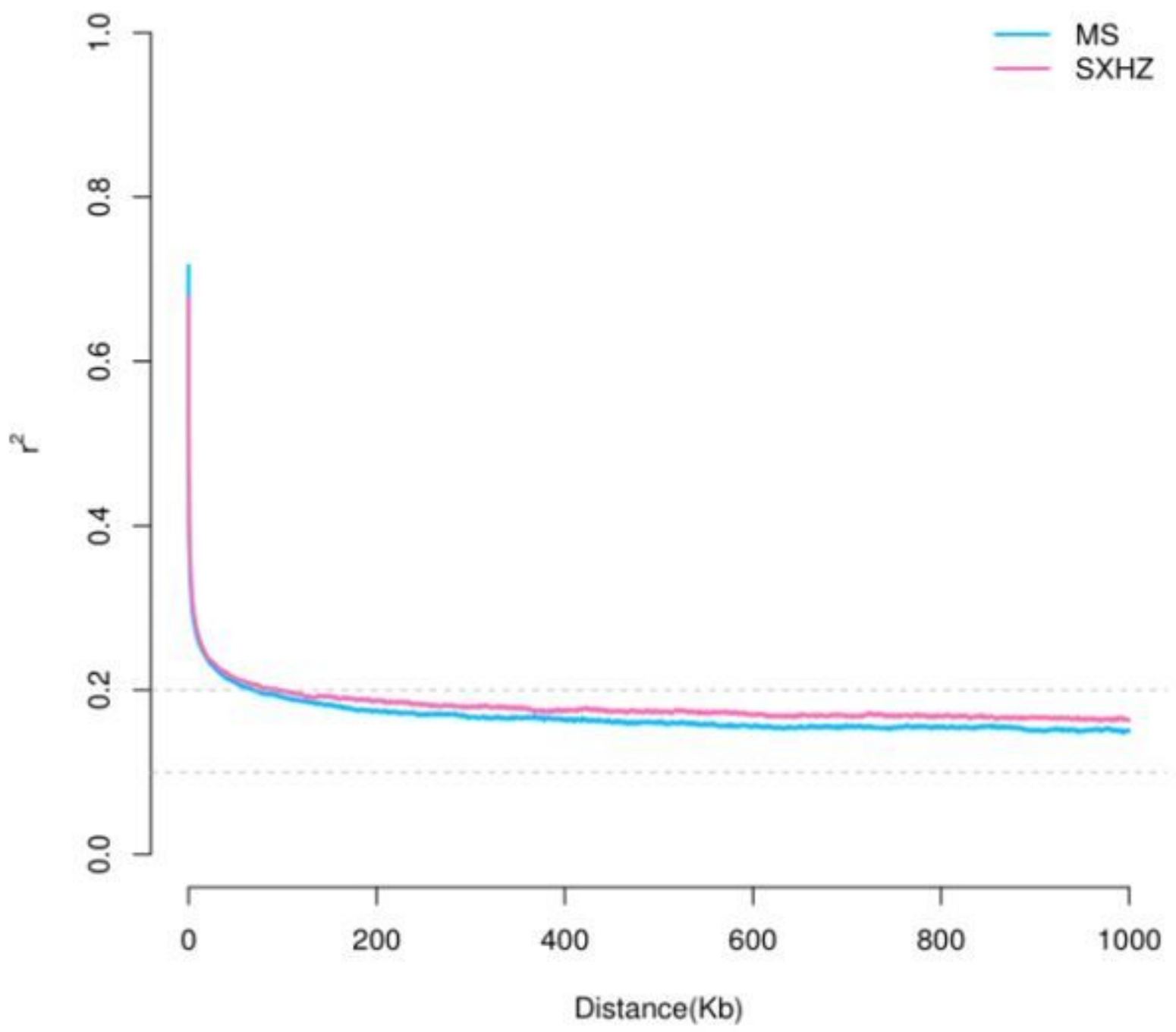

Figure 6

Note:The red line is the LD attenuation curve of Shenxian pigs, and the blue line is the LD attenuation curve of big white pigs. LD attenuation diagram of the Shenxian pig and the Meishan pig populations 


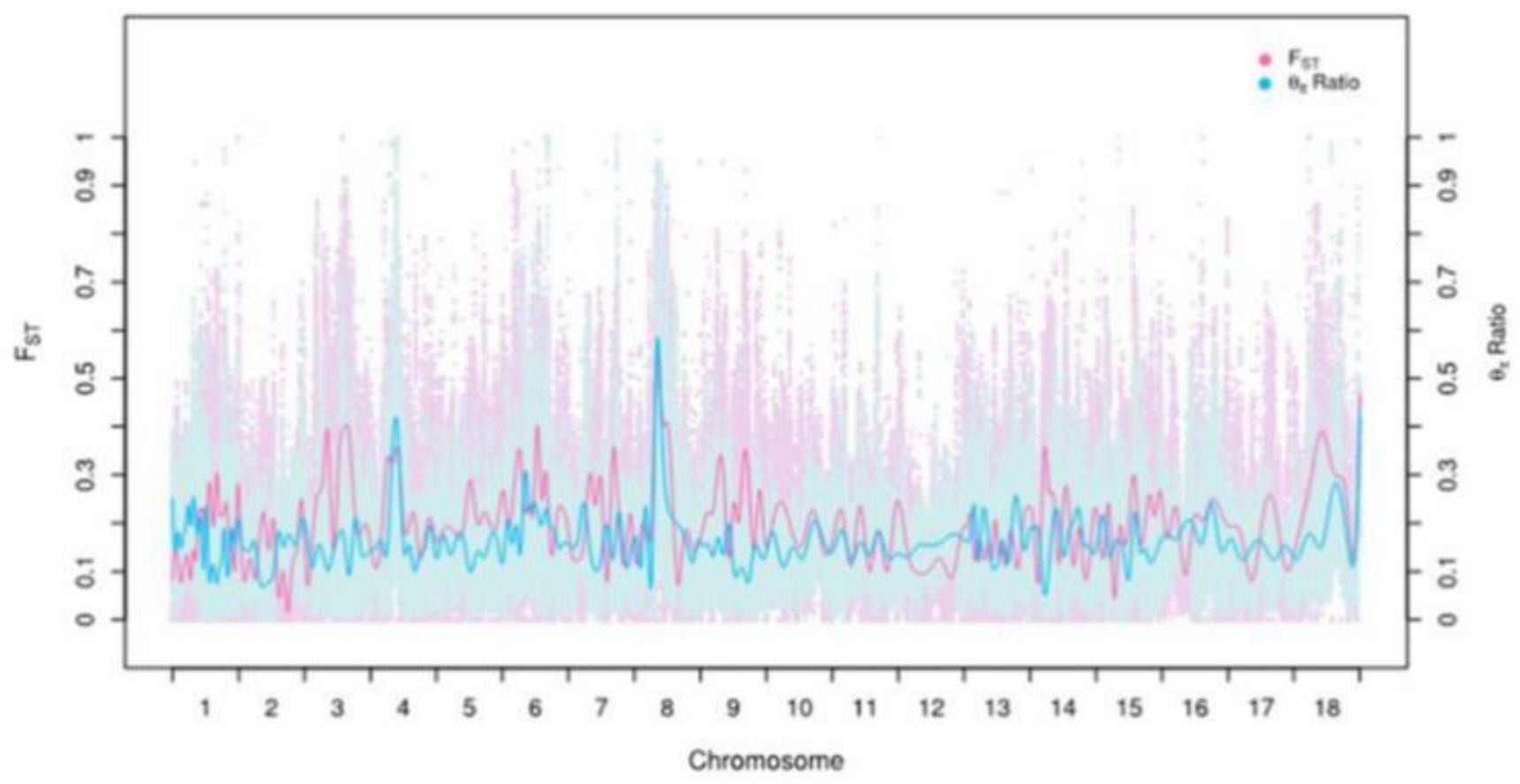

Figure 7

Note: Figure shows the results of Fst and $\theta \pi$ following the completions of size conversions; Red represents the results of the Fst and blue represents the results of $\theta \pi$ ratio. Fst and $\theta \pi$ Manhattan Plot

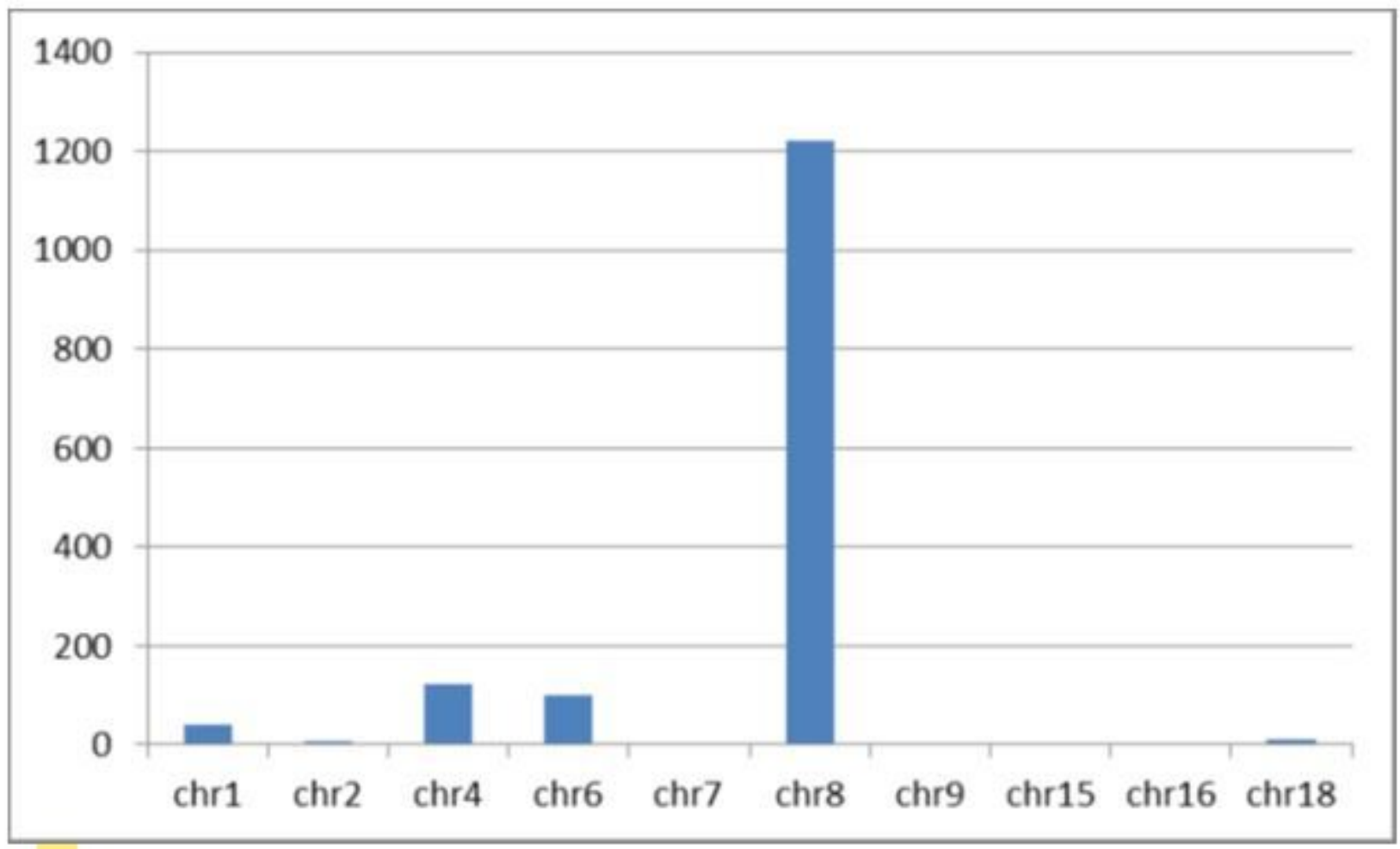

Figure 8 
Note: In the figure, the abscissa represents the chromosome number; and the ordinate represents the number of selected sites. Distribution of the variations on the chromosomes

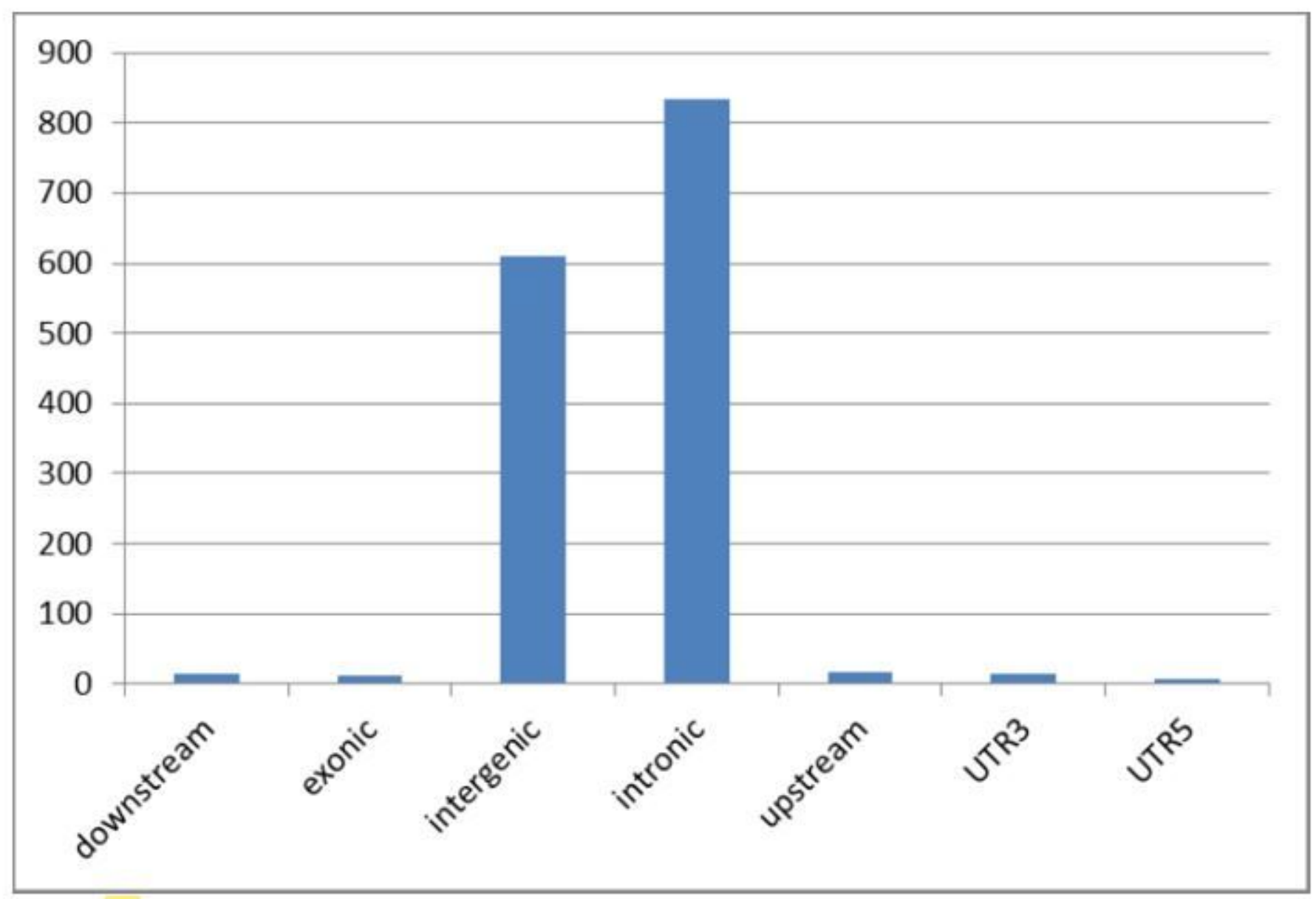

\section{Figure 9}

Note: In the figure, the abscissa represents the gene region; and the ordinate represents the number of selected sites. Distribution of the variation locations 


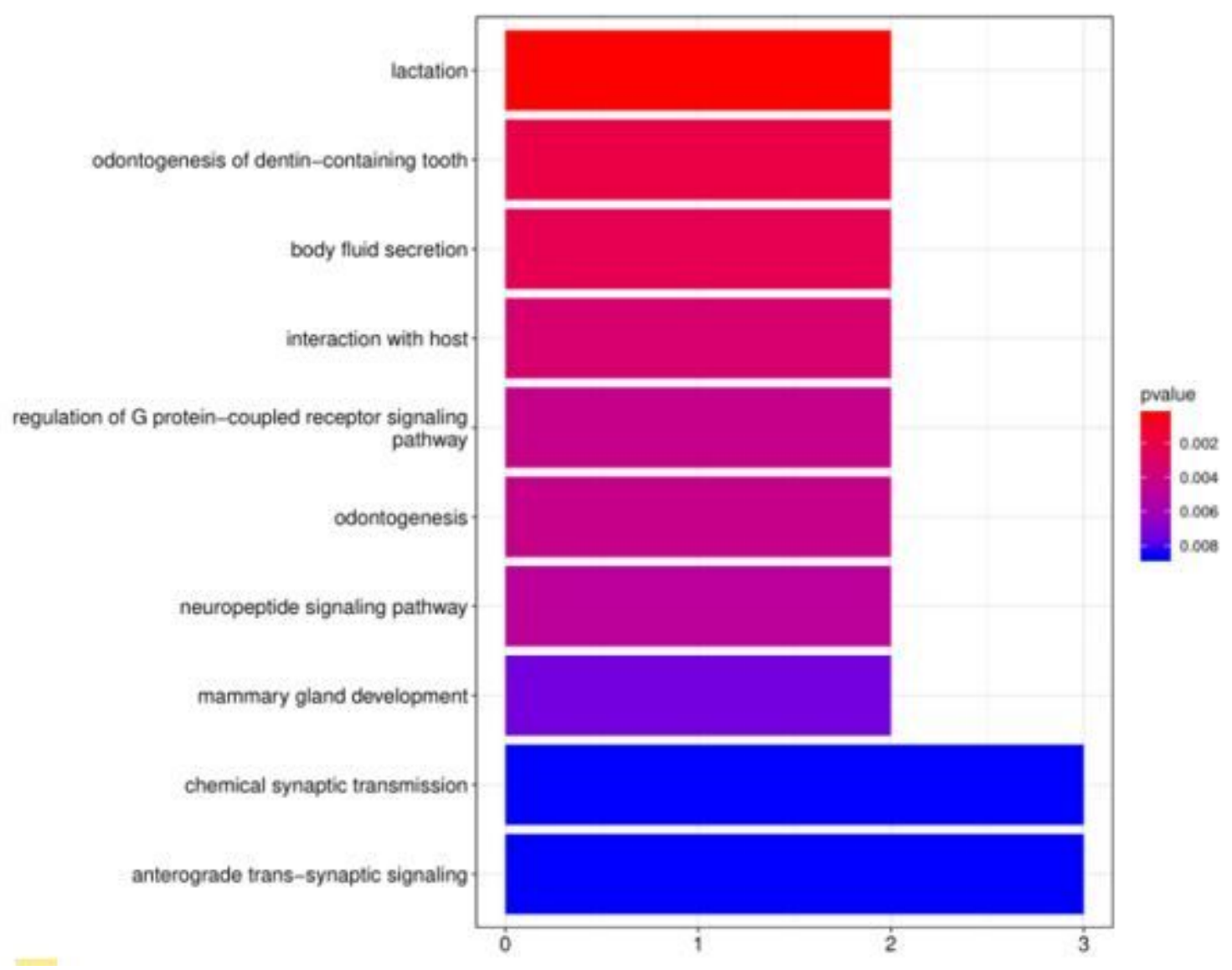

Figure 10

Note: In the figure, the abscissa represents the number of enriched genes; and the ordinate represents the biological processes of enrichment. Enrichment analysis results of the gene biological processes 


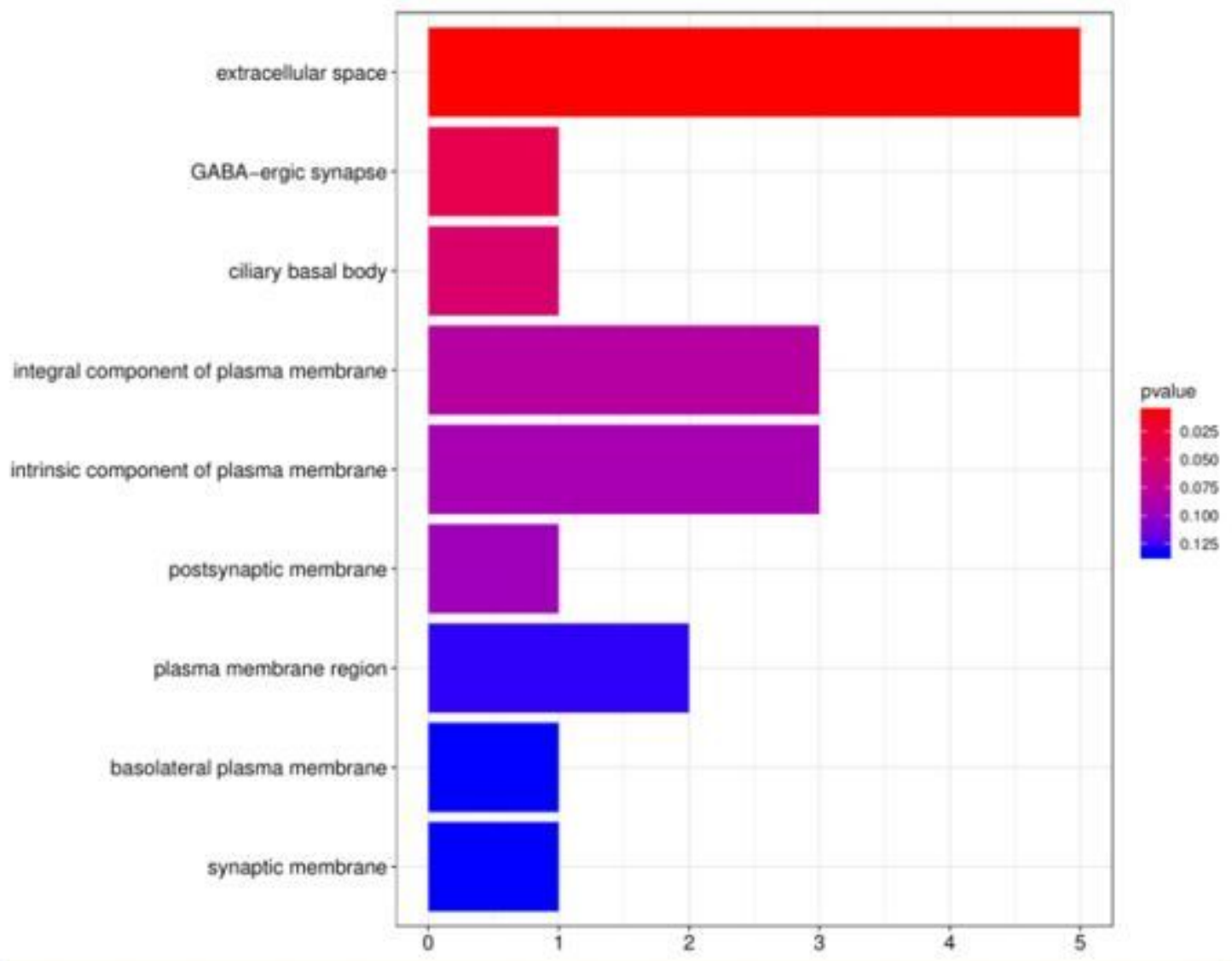

\section{Figure 11}

Note: In the figure, the abscissa represents the number of enriched genes; and the ordinate represents the cell compositions. Enrichment analysis results of the gene cell compositions 


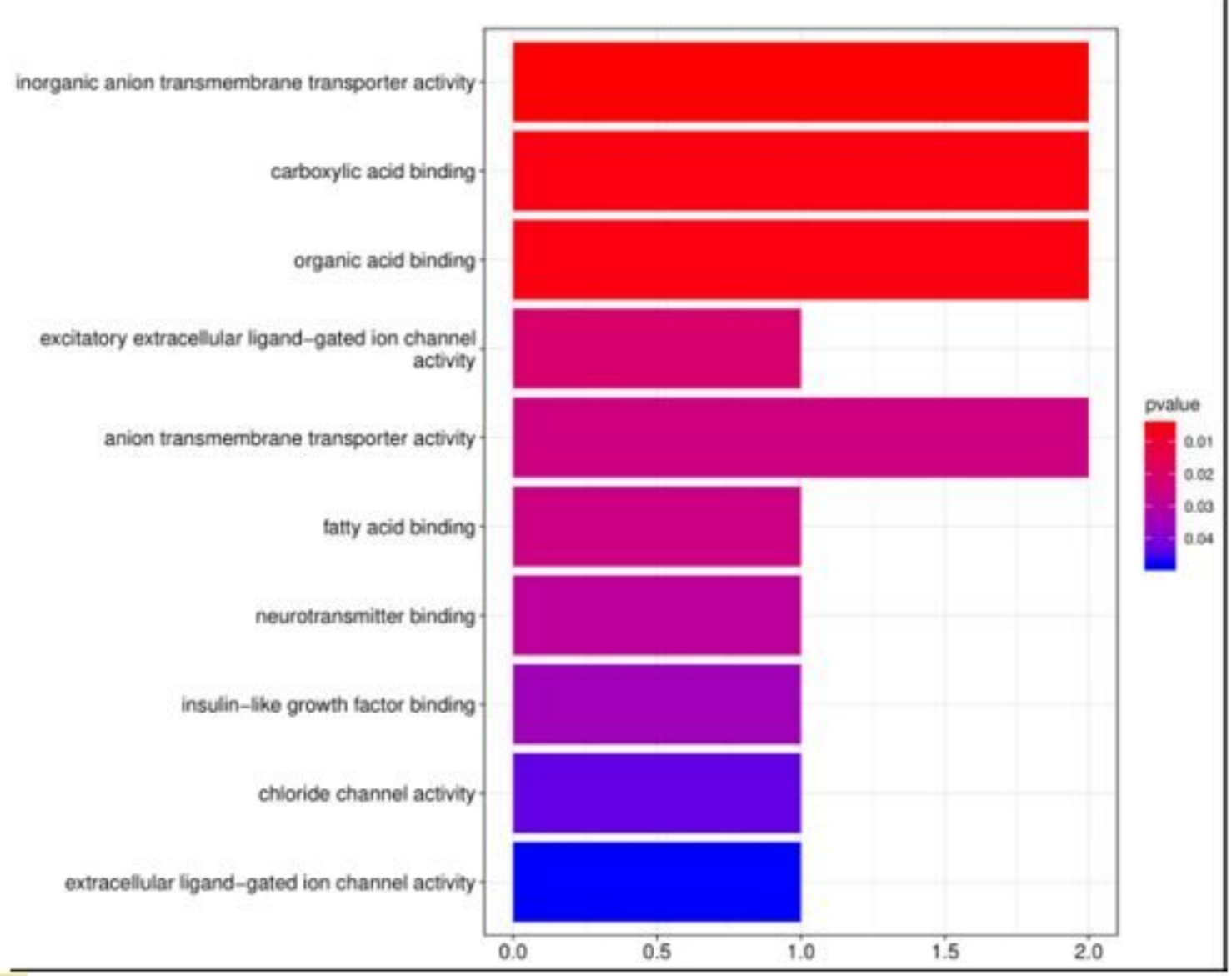

\section{Figure 12}

Note: In the figure, the abscissa represents the number of enriched genes; and the ordinate represents the molecular enriched functions. Enrichment analysis results of the gene molecular functions

Neuroactive ligand-receptor interaction-

Phospholipase D signaling pathway-

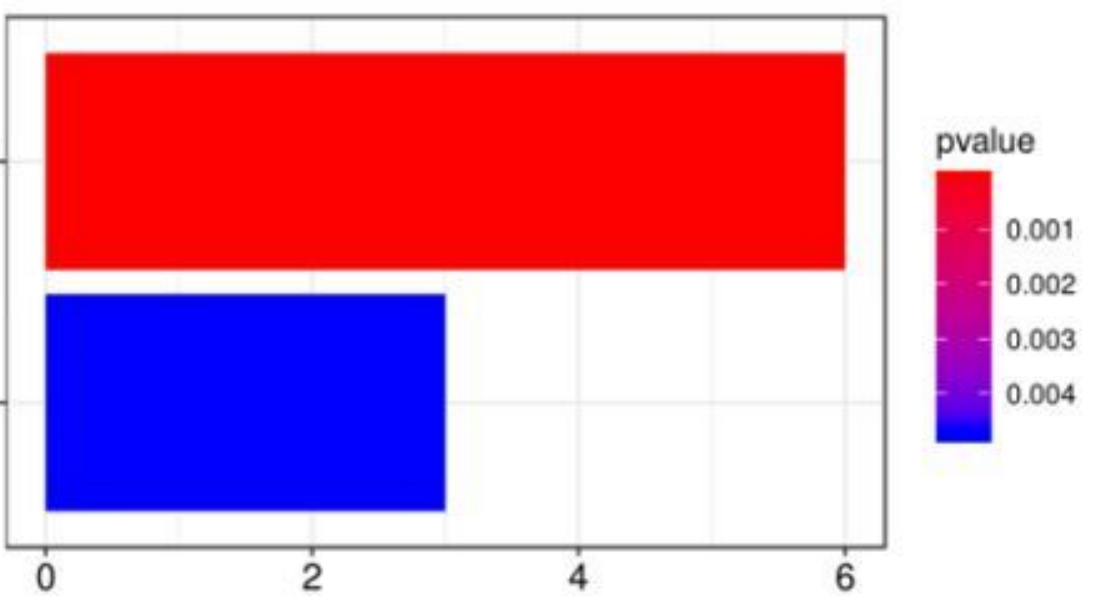

Figure 13 
Note: In the figure, the enriched KEGG pathway terms include the horizontal axis representing the number of genes, and the vertical axis representing the enriched pathways. KEGG pathway analysis results

\section{Supplementary Files}

This is a list of supplementary files associated with this preprint. Click to download.

- AuthorChecklistFull.pdf 